\title{
Dust and self-similarity for the Smoluchowski coagulation equation
}

\section{Poussière et auto-similarité pour l'équation de coagulation de Smoluchowski}

\author{
M. Escobedo ${ }^{\mathrm{a}, 1}$, S. Mischler ${ }^{\mathrm{b}, *}$ \\ a Departamento de Matemáticas, Universidad del País Vasco, Apartado 644, Bilbao E 48080, Spain \\ ${ }^{\mathrm{b}}$ Ceremade, Université Paris IX-Dauphine, place du $M^{a l}$ DeLattre de Tassigny, Paris 75016, France
}

Received 25 November 2004; received in revised form 19 May 2005; accepted 25 May 2005

Available online 28 November 2005

\begin{abstract}
Smoluchowski coagulation equation for a class of homogeneous coagulation rates of degree $\lambda \in[0,2)$. On the one hand for any initial datum $f_{\text {in }} \in \dot{L}_{1}^{1}$ we build a weak solution which conserves the mass when $\lambda \leqslant 1$ and loses mass in finite time (gelation phenomenon) when $\lambda>1$. We then extend this existence result to a measure framework allowing dust source term. In that case we prove that the income dust instantaneously aggregates and the solution does not contain dust phase. On the other hand, we investigate the qualitative properties of self-similar solutions to the Smoluchowski's coagulation equation when $\lambda<1$. We prove regularity results and sharp uniform small and large size behaviour for the self-similar profiles.
\end{abstract}

๑ 2006 L'Association Publications de l'Institut Henri Poincaré. Published by Elsevier B.V. All rights reserved

\section{Résumé}

Nous considérons l'équation de Smoluchowski pour une classe de taux homogènes de degré $\lambda \in[0,2)$. D'une part, pour toute donnée initiale $f_{\text {in }} \in \dot{L}_{1}^{1}$ nous construisons une solution qui conserve la masse lorsque $\lambda \leqslant 1$ et qui perd de la masse en temps fini (phénomène de gélification) lorsque $\lambda>1$. Nous étendons ensuite ce résultat à un contexte mesure qui permet de prendre en compte un terme de source "poussière ». Dans ce cas, nous démontrons que la poussière entrant dans le système s'agglomère instantanément et que la solution ne contient pas de phase poussière. D'autre part, nous étudions les propriétés qualitatives des solutions auto-similaires lorsque $\lambda<1$. Nous démontrons des résultats de régularité et établissons des estimations uniformes sur le comportement du profil auto-similaire pour les petites et les grandes tailles de particules.

(C) 2006 L'Association Publications de l'Institut Henri Poincaré. Published by Elsevier B.V. All rights reserved

MSC: 82C40; 82C05; 82C22; 45K05

Keywords: Regularization property; Self-similar profile; Moment estimates; Large and small size behaviour; Dirac mass source term; Instantaneous aggregation; Smoothness; Bootstrap

\footnotetext{
* Corresponding author. Tel.: +(33) 1440543 60; fax: +(33) 144054036.

E-mail addresses: mtpesmam@lg.ehu.es (M. Escobedo), mischler@ceremade.dauphine.fr (S. Mischler).

1 Tel.: +(34) 946012 649; fax: +(34) 946012516.
} 


\section{Introduction}

The Smoluchowski coagulation equation is a well known model used to describe the dynamics of a system of particles undergoing aggregation events. The present paper is devoted to the analysis of such a model both in the free regime and in a forced regime, in which matter is added to the system by means of a source term. More precisely, if we denote by $f(t, y) \geqslant 0$ the density of particles with mass $y \in \mathbb{R}_{+}:=[0, \infty)$ at time $t \geqslant 0$, the spatially homogeneous coagulation equation with source term is

$$
\begin{aligned}
& \frac{\partial f}{\partial t}(t, y)=Q(f)(t, y)+s(t, y) \quad \text { in } \mathbb{R}_{+}^{2}, \\
& f(0, \cdot)=f_{\text {in }} \quad \text { in } \mathbb{R}_{+},
\end{aligned}
$$

where the coagulation operator $Q$ models the growth mechanism resulting from the encounter of two mother particles and the source term $s$ takes into account the addition of matter to the system. The aggregation mechanism may be schematically written as

$$
\{y\}+\left\{y^{\prime}\right\} \stackrel{a}{\longrightarrow}\left\{y^{\prime \prime}\right\} \quad \text { with } y^{\prime \prime}=y+y^{\prime},
$$

where $a=a\left(y, y^{\prime}\right)$ is the rate of occurrence of the aggregation of two particles of mass $y$ and $y^{\prime}$. The operator $Q$ is then given by

$$
\left\{\begin{array}{l}
Q(f)=Q^{+}(f)-Q^{-}(f), \quad Q^{+}(f)(y)=\frac{1}{2} \int_{0}^{y} a\left(y-y^{\prime}, y^{\prime}\right) f\left(y-y^{\prime}\right) f\left(y^{\prime}\right) \mathrm{d} y^{\prime}, \\
Q^{-}(f)(y)=\int_{0}^{\infty} a\left(y, y^{\prime}\right) f(y) f\left(y^{\prime}\right) \mathrm{d} y^{\prime} .
\end{array}\right.
$$

We refer to the books and review papers of F. Leyvraz [27], P. Laurençot and S. Mischler [23], D.J. Aldous [1], J.H. Seinfeld [39], S.K. Friedlander [17] and R.L. Drake [11] for a basic physical description and motivations and an overview of available mathematical results on coagulation models as well as to the references therein for a more precise physical and mathematical analysis.

In this paper, we assume that the coagulation rate $a$ is a homogeneous function of $y$ and $y^{\prime}$ of degree $\lambda$, i.e. $a\left(r y, r y^{\prime}\right)=r^{\lambda} a\left(y, y^{\prime}\right)$ for all $r>0$. More precisely, we assume

$$
a\left(y, y^{\prime}\right)=y^{\alpha}\left(y^{\prime}\right)^{\beta}+y^{\beta}\left(y^{\prime}\right)^{\alpha}, \quad-1 \leqslant \alpha \leqslant \beta \leqslant 1, \lambda:=\alpha+\beta \in[0,2],(\alpha, \beta) \neq(0,1) .
$$

Our results are still valid for linear combinations of several such rates and also for coagulation rates of the form:

$$
a\left(y, y^{\prime}\right)=\left(y^{v}+\left(y^{\prime}\right)^{v}\right)^{\mu}\left(y^{\sigma}+\left(y^{\prime}\right)^{\sigma}\right), \quad v \mu+\sigma \in[0,1)
$$

(see also [16]). An important particular case is the Smoluchowski's rate $a_{S}$ introduced in [41] which is defined choosing $v=1 / 3, \mu=1$ and $\sigma=-1 / 3$ in (1.5). In order to obtain our results in that case one has to take $\alpha=-1 / 3$, $\beta=1 / 3$ in the statement of the theorems below. Nevertheless, for the sake of brevity, we only write the detailed statements and proofs for the coagulation rates (1.4).

In the Cauchy problem (1.1), (1.2) the initial datum $f_{\text {in }}$ and the source term $s$ are such that $y f_{\text {in }}$ and $y s(t, \cdot)$ are nonnegative measurable functions on $(0, \infty)$ or nonnegative Borel measures on $[0, \infty)$ satisfying (at least)

$$
\int_{0}^{\infty} y \mathrm{~d} f_{\text {in }}(y)<\infty \text { and } \sup _{t>0} \int_{0}^{\infty} y s(t, \mathrm{~d} y)<\infty .
$$

The most fundamental property of this equation is the formal conservation of mass:

$$
\forall T>0 \quad \int_{0}^{\infty} y f(T, \mathrm{~d} y)=\int_{0}^{\infty} y f_{\text {in }}(\mathrm{d} y)+\int_{0}^{T} \int_{0}^{\infty} y s(t, \mathrm{~d} y) .
$$


This mass conservation property is proved to be true for the solutions of the Cauchy problem (1.1), (1.2) for a coagulation rate satisfying (1.4) with $\lambda \leqslant 1$ while it breaks down in finite time (gelation phenomenon) for a coagulation rate satisfying (1.4) with $\lambda>1$ and $s \equiv 0$ (see point (3) just below).

Let us emphasise first that we are specially concerned with the case where $y f_{\text {in }}$ and $y s(t, \cdot)$ contain a Dirac mass (for example $y f_{\text {in }}=\sigma_{\text {in }} \delta_{y=0}$ and $\left.y s(t, \cdot)=\sigma(t) \delta_{y=0}\right)$. This corresponds to the physical situation where part of the matter which constitutes the system (or in other words, part of the mass of the system) is not in the particle phase but in the, so-called, dust phase, i.e. the portion of mass contained in the zero size particles is positive. Dust can be introduced initially in the system (in the initial datum) or can be injected along the evolution of the system (thanks to the source term). When all the mass in the system is contained in the dust phase we have $y f(t, y)=\omega(t) \delta_{y=0}$ whereas when all the system is constituted of particles $y f(t, y)$ does not charge the origin (for instance it is an $L^{1}$ function). In other words, from a mathematical point of view, a system containing some dust is simply described by a density $f(y)$ such that $y f(y)$ charges the origin, i.e. $y f(y) \geqslant \omega \delta_{y=0}$ for some $\omega>0$.

It is very classical in growth models (in the largest sense) to consider physical situations where two phases coexist. Let us give some examples.

(1) The fragmentation equation, which models a linear instantaneous breakage mechanism of particles of positive size, may generate dust in finite time when the fragmentation rate is strong enough (if the rate of fragmentation is singular for small sizes). It is the shattering phenomenon described for instance in $[30,2]$ and the references therein.

(2) The Lifshitz-Slyozov equation [28] provides a model of exchange of matter contained in two different phases (vapour and liquid or liquid and solid for instance): a bath of "elementary particles" (which corresponds to the dust phase here) and particles immersed in the bath. The underlying physical mechanisms are the evaporation and the condensation.

(3) It is commonly accepted (see for instance the pioneer paper by Leyvraz [26] and the review paper [27]) that the Smoluchowski coagulation equation provides a model of finite time phase transition. More precisely, for a coagulation rate $a$ given by (1.4) with $\lambda \in(1,2]$ it has been shown in $[26,18,13]$ that particles (of finite size) aggregate so quickly that particles of infinite size (the gel) are created in finite time: that is the gelation phenomenon.

To our knowledge, there are very few works on Smoluchowski coagulation equation involving dust phase. We have only quoted two papers. On the one hand, the mathematical paper [15] by Fournier and Giet in which the authors consider the coagulation-fragmentation equation with singular fragmentation rate. For such a model dust is a priori produced by the strong fragmentation mechanism (see point (1) above) and then has also to be taken into account in the coagulation mechanism. On the other hand, the physical paper [12] by Duffa and Nguyen-Bui, where the authors are interested in modelling the formation of soot from smoke produced by combustion. In such a situation, the fire generates smoke (elementary benzene molecules) which immediately transforms (by instantaneous aggregation) in soot (particles constituted of more than an hundred of benzene molecules) which in turn follows a standard coagulation mechanism. The authors then describe the system by the density of soot particles, whose growth dynamic is determined by the Smoluchowski coagulation equation, and the smoke as a source term $s$ of $d u s t$ with therefore $y s(t, \mathrm{~d} y)=\sigma(t) \delta_{y=0}$.

There are a great number of physical situations (combustion, aerosols, ...) where matter is added to a system of particles by the mean of dust, i.e. "elementary particles" whose typical size is very small with respect to the size of observable particles. Physical modelling of such a situation is considered in [17,29,7,36] among others. In these works the Smoluchowski coagulation equation with (singular) source term is extensively used. The way to take into account the new matter is to consider a source term $s$ such that $y s(t, \mathrm{~d} y)=\sigma(t) \phi(y)$ where $\phi$ correspond to the distributional density of new matter and the simplest choice is to consider $\phi(y)=\delta_{y=y_{0}}$. Arguing that very small particles are very unstable the authors take $y_{0}>0$. This choice greatly simplifies the mathematical study, although the precise choice of $y_{0}$ is not always clearly determined.

In the first part of this work we consider the Cauchy problem for Eq. (1.1). We revisit the Cauchy problem with non negative measurable initial data $f_{\text {in }}$ satisfying (1.6) and $s \equiv 0$. We prove a regularising effect of the coagulation equation near the origin and give some new estimates on the long and short time behaviour of the moments of the solutions when $\lambda \in[0,1)$. 
We next consider the case of the Cauchy problem for Eq. (1.1) with a source term $s$ and initial datum $f_{\text {in }}$ in a measure framework and such that (1.6) holds. Measure solution is commonly considered in mathematical studies, see for instance [37], while measure solution allowing dust in considered in [15]. Our main result is that dust, which may be present in the initial condition or added to the system thanks to the source term, becomes instantaneously part of the particle phase.

We finally consider the self similar solutions problem for Eq. (1.1) with $s \equiv 0$ and $\lambda \in[0,1)$. The self similar solutions are particular solutions which are invariant by some scaling transformation which depends on the homogeneity $\lambda$ of the coagulation rate $a$. More precisely these solutions are exactly those which are invariant by the preserving mass scaling

$$
f(t, y) \rightarrow \mu^{\frac{2}{1-\lambda}} f\left(\mu t, \mu^{\frac{1}{1-\lambda}} y\right) .
$$

As a consequence a self-similar solution has the form

$$
F(t, y)=t^{-\frac{2}{1-\lambda}} G\left(y t^{-\frac{1}{1-\lambda}}\right),
$$

where the so-called self-similar profile $G$ satisfies the self-similar profile equation

$$
D G+(1-\lambda) Q(G)=0,
$$

and where $D$ is the following linear transport operator preserving the mass

$$
D g=2 g+y \partial_{y} g \text {. }
$$

An other immediate consequence is that any self similar solution $F$ is mass preserving:

$$
\forall t>0 \quad \int_{0}^{\infty} y F(t, y) \mathrm{d} y=\int_{0}^{\infty} y G(y) \mathrm{d} y=: \rho .
$$

As it was shown by van Dongen and Ernst in [10], the function $B y^{-1-\lambda}$ for some normalisation positive constant $B$, is a self-similar solution of the coagulation equation but has no finite mass. When $\lambda=1$ the self similar solutions have a different form. When $\lambda>1$, gelation occurs and such self-similar solutions do not exist.

Notice that all the mass of the self similar solutions is initially in the dust phase. Namely there holds:

$$
F(t, y) y \underset{t \rightarrow 0}{\longrightarrow} \rho \delta_{y=0}
$$

They are therefore particular solutions with initial data containing dust phase. Since no uniqueness result of the solutions of (1.1) with Dirac mass initial condition is known, we do not know whether any solution with Dirac mass initial condition is a self-similar solution.

On the other hand, it is conjectured that the self-similar solution of given mass is unique and that, for a large set of initial data, the corresponding solutions of the coagulation equation behave asymptotically, as $t \rightarrow+\infty$, like the self-similar solution with same mass:

$$
f(t, y) \underset{t \rightarrow \infty}{\sim} F(t, y) .
$$

Explicit self similar solutions are known for the constant kernel $a\left(y, y^{\prime}\right)=1$ (M. von Smoluchowski [40]), for a additive kernel $a\left(y, y^{\prime}\right)=y+y^{\prime}$ and for a multiplicative kernel $a\left(y, y^{\prime}\right)=y y^{\prime}$ (cf. [1] and references therein). For the additive kernel, these solutions were obtained from some hydrodynamic limit of a stochastic model by J. Bertoin in [3]. They were also rederived by G. Menon and R. Pego, and new families of self similar solutions, with no finite mass, for the kernel $a\left(y, y^{\prime}\right)=1$ were obtained by these authors in [31,32]. Moreover for the constant and additive coagulation rate, the asymptotic behaviour of generic solutions, for a large class of initial data, is actually given by the corresponding self-similar solution, see $[31,32,3,19]$ for more details.

Recently the problem of existence of self similar solutions for the coagulation equation has been solved in $[14,16]$ for coagulation rates satisfying (1.4) with $\beta, \lambda \in[0,1)$ : for any given mass $\rho>0$ there exists at least one self-similar profile $G$ which is solution to (1.9), (1.10), (1.11). Our purpose is to prove some regularity and size properties of the self similar profiles.

The behaviour of the self similar solutions of the coagulation equation (assuming their existence) near the origin have been described using formal asymptotics by van Dongen and Ernst in [8,10] and numerically by Lee in [25]. 
- If $\alpha>0$, assuming that the behaviour of the self-similar profile near the origin is "regular" (i.e. the limit $\lim _{x \rightarrow 0} G(a x) / G(x)$ exists for all $a>0$, see [27]), van Dongen and Ernst obtained in [8,10] that

$$
G(y) \sim A y^{-(1+\lambda)}, \quad \text { as } y \rightarrow 0,
$$

for some explicit, positive constant $A$ depending only on $a$.

- If $\alpha=0$, under the same hypothesis, the same authors obtain for a normalised self similar profile $G$, that is $M_{1}(G)=1$, the following asymptotic behaviour

$$
\begin{aligned}
& G(y) \sim y^{-\tau}, \quad \text { as } y \rightarrow 0 ; \\
& \tau=2-(1-\lambda) \int_{0}^{\infty} y^{\lambda} G(y) \mathrm{d} y<1+\lambda .
\end{aligned}
$$

- If $\alpha<0$ the hypothesis of regular behaviour at the origin does not seem to hold. In that case, the same authors obtain in $[8,10]$ :

$$
G(y) \sim A y^{-2} \exp \left(-B y^{-|\alpha|}\right) \quad \text { as } y \rightarrow 0, \quad B=\frac{1}{(1-\lambda)|\alpha|} \int_{0}^{\infty} y^{\lambda-\alpha} G(y) \mathrm{d} y .
$$

The behaviour of the self similar profiles as $y \rightarrow+\infty$ has been treated by van Dongen and Ernst in [9] when $\beta=1$ and in [10] when $\beta \in[0,1)$. They establish

$$
G(y) \sim A y^{-\lambda} \mathrm{e}^{-\delta y} \quad \text { as } y \rightarrow \infty
$$

where $A$ and $\delta$ are two positive constants related with the coagulation rate $a$.

The results obtained in [14] and [16] gave in particular rigorous estimates on some of the moments of the self similar profiles. These estimates give a less precise information on the behaviour of these profiles than those obtained by asymptotic expansions. Although they also indicate that the behaviour, near the origin, is more regular in the case $\alpha<0$ than for $\alpha \geqslant 0$. In the present work, we improve the previous rigorous estimates on the profiles in two ways: we obtain lower and upper bounds of the profiles as $y \rightarrow 0$ and $y \rightarrow+\infty$, and we obtain regularity results for these profiles. Nevertheless our results are still far from (1.14)-(1.17).

Let conclude this introduction by some fundamental and well known remarks. A self similar profile $G$ is stationary solution of the equation

$$
\frac{\partial g}{\partial t}=D g+(1-\lambda) Q(g) \quad \text { in } \mathbb{R}_{+} \times \mathbb{R}_{+},
$$

that we call the coagulation equation in self-similar variables. Now, on the other hand, it is straightforward (using the scaling (1.7)) to check that, if $g$ is a solution to (1.18) then the function $f$ defined by

$$
f(t, y):=(1+t)^{-\frac{2}{1-\lambda}} g\left(\frac{\ln (1+t)}{1-\lambda}, y(1+t)^{-\frac{1}{1-\lambda}}\right)
$$

is a solution to the coagulation equation (1.1) with same initial datum and $s \equiv 0$. A immediate and useful consequence is the following relation between the power moments of $f$ and $g$ :

$$
M_{k}(f(t, \cdot))=(1+t)^{\frac{k-1}{1-\lambda}} M_{k}\left(g\left(\frac{\ln (1+t)}{1-\lambda}, \cdot\right)\right),
$$

where for any measurable function $h: \mathbb{R}_{+} \rightarrow \mathbb{R}_{+}$and any real $k \in \mathbb{R}$ we have defined the moment of $h$ of order $k$ by

$$
M_{k}(h)=\int_{0}^{\infty} h(y) y^{k} \mathrm{~d} y .
$$

Reciprocally, if $f$ satisfies the coagulation equation (1.1), we obtain a solution $g$ to (1.18) defining

$$
g(t, y)=\mathrm{e}^{2 t} f\left(\mathrm{e}^{(1-\lambda) t}-1, y \mathrm{e}^{t}\right) .
$$


We have therefore an equivalence between (1.18) and (1.1) with $s \equiv 0$ thanks to the simple change of variables (1.21) and (1.19). As we will see, in order to establish properties on the solution of the coagulation equation (1.1), it is often more convenient to work on the coagulation in self-similar variables (1.18) than to work directly on the coagulation equation (1.1) itself.

The paper is organised as follows. In Section 2 we state the precise definitions of solution we will deal with and we state our main results on the Cauchy problem and on the profile problem. In Section 3 we establish some (somewhat new) moment estimates. In Section 4 we prove regularity and uniform by above estimates while in Section 5 the uniform by below estimates are proved. Finally, in Section 6 we gather a priori estimates on the solution of Eq. (1.1) and give a sketch of the proof of the existence of solutions to the Cauchy problem.

\section{Main results}

We first recall some well known elementary but fundamental formal computations in order to give a weak sense to the coagulation operator (1.3). We mean by weak sense a formulation of $Q(f)$ in which the terms $Q^{ \pm}(f)$ are not necessarily well defined separately but the coagulation term makes sense due to cancellations. This will motivate the definitions of weak solutions introduced in Definition 2.3 and Definition 2.10 below.

On the one hand, for any functions $f$ and $\phi$, the following key identities formally hold

$$
\begin{aligned}
\int_{0}^{\infty} Q(f) \phi \mathrm{d} y & =\frac{1}{2} \int_{0}^{\infty} \int_{0}^{y} a\left(y-y^{\prime}, y^{\prime}\right) f\left(y-y^{\prime}\right) f\left(y^{\prime}\right) \phi(y) \mathrm{d} y^{\prime} \mathrm{d} y-\int_{0}^{\infty} \int_{0}^{\infty} a\left(y, y^{\prime}\right) f(y) f\left(y^{\prime}\right) \phi(y) \mathrm{d} y^{\prime} \mathrm{d} y \\
& =\frac{1}{2} \int_{0}^{\infty} \int_{0}^{\infty} a f f^{\prime}\left[\phi^{\prime \prime}-\phi-\phi^{\prime}\right] \mathrm{d} y^{\prime} \mathrm{d} y \\
& =\int_{0}^{\infty} \int_{0}^{\infty} y^{\alpha}\left(y^{\prime}\right)^{\beta} f f^{\prime}\left[\phi^{\prime \prime}-\phi-\phi^{\prime}\right] \mathrm{d} y^{\prime} \mathrm{d} y=:\langle\mathcal{Q}(f), \phi\rangle,
\end{aligned}
$$

where we have first performed the change of variables $\left(y, y^{\prime}\right) \rightarrow\left(z=y-y^{\prime}, y^{\prime}\right)$ in the first term of $Q(f)$ and the symmetry of $a f f^{\prime}$ in the second term, and we have next used the symmetry of $f f^{\prime}\left[\phi^{\prime \prime}-\phi-\phi^{\prime}\right]$. Here and below we use the notations $\varphi=\varphi(y), \varphi^{\prime}=\varphi\left(y^{\prime}\right), \varphi^{\prime \prime}=\varphi\left(y^{\prime \prime}\right), y^{\prime \prime}=y+y^{\prime}$ for any $y, y^{\prime} \in \mathbb{R}_{+}$and any function $\varphi$.

On the other hand, for any functions $f$ and $\psi$ the following identities formally hold, starting from (2.1) with $\phi=y \psi$,

$$
\begin{aligned}
\int_{0}^{\infty} Q(f) y \psi \mathrm{d} y & =\frac{1}{2} \int_{0}^{\infty} \int_{0}^{\infty} a f f^{\prime}\left(y \psi^{\prime \prime}+y^{\prime} \psi^{\prime \prime}-y \psi-y^{\prime} \psi^{\prime}\right) \mathrm{d} y \mathrm{~d} y^{\prime} \\
& =\int_{0}^{\infty} \int_{0}^{\infty} a f f^{\prime} y\left\{\psi^{\prime \prime}-\psi\right\} \mathrm{d} y \mathrm{~d} y^{\prime} \\
& =\int_{0}^{\infty} \int_{0}^{\infty} a y f f^{\prime}\left\{\int_{y}^{y+y^{\prime}} \partial_{z} \psi(z) \mathrm{d} z\right\} \mathrm{d} y \mathrm{~d} y^{\prime}=\int_{0}^{\infty} \partial_{z} \psi(z)\left\{\int_{0}^{\infty} \int_{0}^{\infty} \mathbf{1}_{y \leqslant z \leqslant y+y^{\prime}} a y f f^{\prime} \mathrm{d} y \mathrm{~d} y^{\prime}\right\} \mathrm{d} z \\
& =-\int_{0}^{\infty} \partial_{z}(\mathcal{C}(f))(z) \psi(z) \mathrm{d} z,
\end{aligned}
$$

with

$$
\mathcal{C}(f)(z):=\int_{0}^{z} f y\left\{\int_{z-y}^{\infty} a f^{\prime} \mathrm{d} y^{\prime}\right\} \mathrm{d} y
$$




$$
=\left(y^{\alpha+1} f\right) \star_{z} L_{\beta}(f)+\left(y^{\beta+1} f\right) \star_{z} L_{\alpha}(f) .
$$

Here we have defined for any functions $g$ and $h$ the modified convolution operation by

$$
z \mapsto h \star_{z} g:=\int_{0}^{z} h(y) g(z-y) \mathrm{d} y,
$$

and we have set

$$
L_{v}(f)(u):=\int_{u}^{\infty} v^{v} f(v) \mathrm{d} v .
$$

In order to give a rigorous meaning to these different formulations of the coagulation operator we need some notations. We denote by $L_{\text {loc }}^{1}$ the space of measurable functions $f:(0, \infty) \rightarrow \mathbb{R}$, such that $f \in L^{1}\left(R^{-1}, R\right)$ for any $R>1$ and by $M_{\mathrm{loc}}^{1}$ the space of measures with the corresponding property. For any given continuous function $\varphi:(0, \infty) \rightarrow(0, \infty)$, we define

$$
M_{\varphi}^{1}:=\left\{f \in M_{\mathrm{loc}}^{1}, \mathcal{M}_{\varphi}(|f|)<\infty\right\}, \quad L_{\varphi}^{1}:=M_{\varphi}^{1} \cap L_{\mathrm{loc}}^{1},
$$

where the generalised moment $\mathcal{M}_{\varphi}$ is defined by

$$
\mathcal{M}_{\varphi}(f):=\int_{0}^{\infty} \varphi(y) \mathrm{d} f(y) .
$$

All these are Banach spaces. In particular, we may define the usual weak convergence in $L_{\varphi}^{1}$ (resp. $M_{\varphi}^{1}$ ) and then the space $C\left(I ; L_{\varphi}^{1}\right.$-weak) (resp. $C\left(I ; M_{\varphi}^{1}\right.$-weak)) for a given interval of time $I$. For instance we say that $f \in C\left(I ; M_{\varphi}^{1}\right.$-weak) if $f(t) \in M_{\varphi}^{1}$ for any $t \in I$ and for any $\chi \in C_{b}([0, \infty)$, the space of continuous and bounded functions, the function $t \mapsto \mathcal{M}_{1}(f \varphi \chi)$ is continuous. Similarly, we write $f \in C\left(I ; L_{\text {loc }}^{1}\right.$-weak $)$ in order to express that $f(t) \in L_{\text {loc }}^{1}$ for any $t \in I$ and for any $\chi \in L^{\infty}$ with compact support in ]0, $\infty$ [ the function $t \mapsto \mathcal{M}_{1}(f \chi)$ is continuous. In order to shorten notations we also (abusively) denote $M_{k}=\mathcal{M}_{y^{k}}, \dot{L}_{k}^{1}=L_{y^{k}}^{1}, \dot{M}_{k}^{1}=M_{y^{k}}^{1}, L_{k}^{1}=L_{1+y^{k}}^{1}$ and $M_{k}^{1}=M_{1+y^{k}}^{1}$, for any $k \in \mathbb{R}$.

\section{Lemma 2.1.}

(i) For any $f \in \mathcal{X}_{a}^{\prime}$ with $\mathcal{X}_{a}^{\prime}:=\dot{M}_{\alpha+1}^{1}+\dot{M}_{\beta}^{1}$ if $\beta \leqslant 1+\alpha$ and $\mathcal{X}_{a}^{\prime}:=\dot{M}_{\alpha+1}^{1} \cap \dot{M}_{\beta}^{1}$ if $\beta \geqslant 1+\alpha$ the operator $\mathcal{Q}(f)$ is well defined in $\mathcal{D}^{\prime}(0, \infty)$. More precisely, for any compact set $K \subset(0, \infty)$, there exists a constant $C_{K}$ such that for any $f \in \mathcal{X}_{a}:=\dot{M}_{1}^{1} \cap \dot{M}_{\min (1, \alpha+1)}^{1} \subset \mathcal{X}_{a}^{\prime}$ and any $\phi \in \mathcal{D}(0, \infty)$ with $\operatorname{supp} \phi \subset K$ there holds

$$
|\langle\mathcal{Q}(f), \phi\rangle| \leqslant C_{K}\|f\|_{\dot{M}_{1}^{1}}\|f\|_{\mathcal{X}_{a}}\|\phi\|_{C^{1}(K)} .
$$

(ii) For any $f \in \dot{M}_{k}^{1}$ with $k \geqslant v$ there holds $L_{v}(f) y^{k-v} \in L^{\infty}$. As a consequence, for any $f \in \dot{M}_{1}^{1}$ if $\alpha>0$ and for any $f \in \dot{M}_{k}^{1} \cap \dot{M}_{1}^{1}$ with $k<1+\alpha$ if $\alpha \leqslant 0$, the operator $\mathcal{C}(f)$ is well defined in $M_{\mathrm{loc}}^{1}$ and $y \mathcal{Q}(f)=-\partial_{y}(\mathcal{C}(f))$ in $\mathcal{D}^{\prime}(0, \infty)$.

(iii) For any $f \in \dot{L}_{\alpha+\min (0, k)}^{1} \cap \dot{L}_{\beta+\max (0, k)}^{1}$ (resp. $\left.f \in \dot{M}_{\alpha+\min (0, k)}^{1} \cap \dot{M}_{\beta+\max (0, k)}^{1}\right)$ with $k \in \mathbb{R}$, there holds $Q^{ \pm}(f) \in$ $L_{k}^{1}\left(\right.$ resp. $\left.Q^{ \pm}(f) \in M_{k}^{1}\right)$, and then $Q(f)=\mathcal{Q}(f)=y^{-1} \partial_{y}(\mathcal{C}(f))$ in $\mathcal{D}^{\prime}(0, \infty)$ for any $f \in \dot{M}_{\alpha}^{1} \cap \dot{M}_{\beta+k}^{1}$ with $k \geqslant 1-\beta$.

Proof of Lemma 2.1. Step 1. Proof of (i). Take $\phi \in C_{c}^{1}(0, \infty)$ and introduce $y_{0}, y_{1} \in(0, \infty)$ such that $\operatorname{supp} \phi \subset$ $K:=\left[2 y_{0}, y_{1}\right]$ and $\Delta_{\phi}:=\phi^{\prime \prime}-\phi-\phi^{\prime}$. From the definition (2.2) of $\langle\mathcal{Q}(f), \phi\rangle$ it will be enough to prove that $A:=$ $y^{\alpha}\left(y^{\prime}\right)^{\beta} f f^{\prime}\left|\Delta_{\phi}\right| \in M^{1}(\Omega), \Omega:=(0, \infty) \times(0, \infty)$, in order to conclude that $\mathcal{Q}(f)$ is well defined. We decompose $\Omega=\Omega_{1} \cup \Omega_{2} \cup \Omega_{3} \cup \Omega_{4}$ with

$$
\begin{aligned}
& \Omega_{1}:=(] 0, y_{0}[\times] 0, y_{0}[) \cup(] 0, y_{0}[\times] y_{1}, \infty[) \cup(] y_{1}, \infty[\times] 0, y_{0}[), \\
& \Omega_{2}=\left[y_{0}, \infty\left[\times\left[y_{0}, \infty\left[, \quad \Omega_{3}=\right] 0, y_{0}[\times] y_{0}, y_{1}\left[, \quad \Omega_{4}=\right] y_{0}, y_{1}[\times] 0, y_{0}[,\right.\right.\right.
\end{aligned}
$$


and we deal with each subset $\Omega_{i}$ separately.

- On $\Omega_{1}$, there holds $\Delta_{\phi} \equiv 0$, and then $A=0$.

- On $\Omega_{2}$, there holds $A \leqslant 2\|\phi\|_{L^{\infty}} y^{\alpha} f\left(y^{\prime}\right)^{\beta} f^{\prime}$.

- On $\Omega_{3}$, we have

$$
\Delta_{\phi}=\phi\left(y+y^{\prime}\right)-\phi\left(y^{\prime}\right)=y \phi^{\prime}\left(y+\theta y^{\prime}\right)
$$

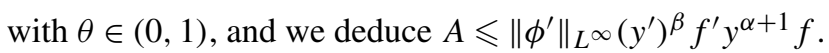

- On $\Omega_{4}$, the same argument as on $\Omega_{3}$ gives $A \leqslant\left\|\phi^{\prime}\right\|_{L^{\infty}} y^{\alpha} f\left(y^{\prime}\right)^{\beta+1} f^{\prime}$.

Gathering the above estimates, we get

$$
A \leqslant C_{K}\|\phi\|_{W^{1, \infty}} \psi_{\alpha} f \psi_{\beta}^{\prime} f^{\prime}, \quad \psi_{\gamma}(y):=y^{\gamma+1} \mathbf{1}_{y \leqslant 1}+y^{\gamma} \mathbf{1}_{y \geqslant 1},
$$

for some constant $C_{K}$ depending of the support $K$ of $\phi$.

For $f \in \mathcal{X}_{a}^{\prime}$, we observe that $\psi_{\alpha} \leqslant \psi_{\alpha, \beta}, \psi_{\beta} \leqslant \psi_{\alpha, \beta}$, where we have defined $\psi_{\gamma, \delta}(y):=y^{\gamma+1} \mathbf{1}_{y \leqslant 1}+y^{\delta} \mathbf{1}_{y \geqslant 1}$, and we deduce from (2.10) that

$$
|\langle\mathcal{Q}(f), \phi\rangle| \leqslant C_{K}\|f\|_{\mathcal{X}_{a}^{\prime}}^{2}\|\phi\|_{C^{1}(K)}
$$

since that $\|f\|_{\mathcal{X}_{a}^{\prime}}=\left\|f \psi_{\alpha, \beta}\right\|_{M^{1}}$

For $f \in \mathcal{X}_{a}$, we observe that $\psi_{\alpha} \leqslant \psi_{\alpha, 1}, \psi_{\beta} \leqslant y$, and we deduce (2.9) from (2.10) again.

Step 2. Proof of (ii). We start remarking that for $g \in \dot{M}_{k}^{1}, k \geqslant v$, we have

$$
\forall z \in \mathbb{R}_{+} \quad L_{\nu}(g)(z)=\int_{z}^{\infty} y^{\nu-k} y^{k} g \mathrm{~d} y \leqslant z^{\nu-k} M_{k}(g) .
$$

Therefore, by the hypothesis on $f$, we have that $L_{\alpha}(f), L_{\beta}(f) \in L_{\text {loc }}^{1}, y^{\alpha+1} f, y^{\beta+1} f$ are two measures locally bounded on $(0,+\infty)$ and the operator $\mathcal{C}(f)$ is well defined as a function of $L_{\text {loc }}^{1}$ (see Lemma 4.4 for details). We easily conclude thanks to the formal computation leading to (2.4) from (2.2), which can be made rigorous thanks to the above assumptions.

Step 3. Proof of (iii). This is a straightforward verification. Notice that when $f$ is a measure, the coagulation operator $Q(f)=Q^{+}(f)-Q^{-}(f)$ is defined by duality on test functions $\varphi \in C_{b}([0, \infty))$ as follows:

$$
\left\langle Q^{+}(f), \varphi\right\rangle=\int_{0}^{\infty} \int_{0}^{\infty} a \varphi^{\prime \prime} \mathrm{d} f(y) \mathrm{d} f\left(y^{\prime}\right), \quad\left\langle Q^{-}(f), \varphi\right\rangle=\int_{0}^{\infty} \int_{0}^{\infty} a \varphi \mathrm{d} f(y) \mathrm{d} f\left(y^{\prime}\right),
$$

and then

$$
\left\langle Q^{ \pm}(f), \varphi\right\rangle \leqslant\|\varphi\|_{L^{\infty}}\left(\int_{0}^{\infty}\left(y^{\alpha}+y^{\beta}\right) \mathrm{d} f(y)\right)^{2} .
$$

Remark 2.2. When $\alpha, \beta \in(0,1), \mathcal{Q}\left(y^{-1-\lambda}\right)$ is well defined since $y^{-1-\lambda} \in \dot{L}_{\alpha+1}^{1}+\dot{L}_{\beta}^{1} \subset \mathcal{X}_{a}^{\prime}$.

Definition 2.3. We say that a function $f: \mathbb{R}_{+}^{2} \rightarrow \mathbb{R}_{+}$is a (global) weak solution to the coagulation equation (1.1) with $s \equiv 0$ if

$$
f \in C(] 0, \infty\left[; L_{\text {loc }}^{1} \text {-weak }\right) \cap L_{\text {loc }}^{\infty}(] 0, \infty\left[; \mathcal{X}_{a}\right),
$$

and

$$
\forall \varphi \in C_{c}^{1}\left(\mathbb{R}_{+}^{2}\right) \quad \int_{0}^{\infty} \int_{0}^{\infty} f(t, y) \partial_{t} \varphi \mathrm{d} y \mathrm{~d} t+\int_{0}^{\infty}\langle\mathcal{Q}(f(t, \cdot)), \varphi(t, \cdot)\rangle \mathrm{d} t=0 .
$$


We say that a function $f: \mathbb{R}_{+}^{2} \rightarrow \mathbb{R}_{+}$is a weak solution to the Cauchy problem (1.1), (1.2) with $s \equiv 0$ and initial datum $0 \leqslant f_{\text {in }} \in \dot{L}_{1}^{1}$ if $f$ is a weak solution to the coagulation equation $(1.1), f \in C\left(\left[0, \infty\left[; L_{\text {loc }}^{1}\right.\right.\right.$-weak $)$ and $f(0, \cdot)=f_{\text {in }}$ a.e.

We finally introduce the space $\mathcal{V}_{\text {in }}$ of admissible initial data, defined by

$$
\mathcal{V}_{\text {in }}:=\bigcup_{m<1}\left(\dot{L}_{1}^{1} \cap \dot{L}_{m}^{1}\right) \quad \text { if } \beta=1, \alpha<0
$$

and

$$
\mathcal{V}_{\text {in }}:=\dot{L}_{1}^{1} \quad \text { if } \beta<1 \text { or if } \beta=1, \alpha>0 .
$$

Concerning the Cauchy problem we have the following result:

Theorem 2.4. Assume (1.4) and $s \equiv 0$. For any $0 \leqslant f_{\text {in }} \in \mathcal{V}_{\text {in }}$ there exists at least one weak solution $f$ to the Cauchy problem (1.1), (1.2). This solution can be built in such a way that

$$
t \mapsto \mathcal{M}_{\varphi}(t), \quad t \mapsto \int_{0}^{\infty} \Lambda(f(t, \cdot)) y^{k} \mathrm{~d} y
$$

are decreasing functions for any subadditive function $\varphi$, for any smooth convex and increasing function $\Lambda$ with $\Lambda(0)=0$ and for $k=0,1$, and furthermore

$$
f(t, \cdot) \rightarrow 0 \quad \text { a.e. when } t \rightarrow \infty \text {. }
$$

Moreover, the following additional properties hold:

Case 1. If $\lambda \in[0,1]$, one can build the solution in such a way that it is mass preserving and it satisfies that the function $t \mapsto M_{k}(t)$ is increasing for $k>1$.

Case 2. If $\lambda, \beta \in[0,1)$, one can build a solution which satisfies $f \in C(] 0, \infty\left[; \dot{L}_{k}^{1}\right)$ for any $k \in I_{a}$, where $I_{a}=(\lambda, 1)$ if $\alpha>0, I_{a}=[\lambda, 1)$ if $\alpha=0$ and $I_{a}=(-\infty, 1)$ if $\alpha<0$, and more precisely, assuming $f_{\text {in }} \in \dot{L}_{1}^{1} \cap \dot{L}_{M}^{1}$ for some $M>1$, there holds

$$
\forall k \in I_{a} \cup[1, M] \quad C_{1, k} t^{\frac{k-1}{1-\lambda}} \leqslant M_{k}(f(t, \cdot)) \leqslant C_{2, k} t^{\frac{k-1}{1-\lambda}} \quad \forall t \geqslant 2,
$$

and

$$
\forall k \in I_{a} \cap[\lambda, 1] \quad M_{k}(f(t, \cdot)) \leqslant C_{3, k} t^{-1} \quad \forall t \in(0,2],
$$

for some positive constants $C_{i, k}$ depending of $f_{\text {in }}$. We refer to Corollary 3.10 for a more precise statement.

Case 3. If $\lambda>1$, gelation occurs in finite time, i.e. there exists $T_{g} \in[0, \infty)$ such that

$$
M_{1}(t) \equiv M_{1}(0) \quad \forall t \in\left[0, T_{g}\right), \quad M_{1}(t)<M_{1}(0) \quad \forall t \in\left(T_{g}, \infty\right) .
$$

\section{Remark 2.5.}

(i) It has already been proved in $[43,38,23,14]$ that for any $f_{\text {in }} \in L_{2 \alpha}^{1} \cap L_{1+\beta}^{1}$ there exists a unique mild solution (in the sense of [14, Definition 2.4]) in $C\left([0, \infty) ; \dot{L}_{2 \alpha}^{1} \cap L_{1+\beta}^{1}\right)$.

(ii) The existence of solutions is the object of many previous references. In the case $\alpha \geqslant 0$ we refer, for instance to [42]. In the case $\alpha<0$, we refer to [37,33]. In any case, we refer to the recent survey [23] and the references quoted therein.

(iii) Gelation has been proved to occur when $\lambda>1$ in [13] for any solution associated to an initial datum $f_{\text {in }} \in L_{1}^{1}$, see also $[26,18]$.

(iv) When $\beta<1$ we may extend the above existence result to an initial datum $f_{\text {in }} \in \dot{L}_{1}^{1}+\dot{L}_{\beta}^{1}$. In that case, we have to replace the bound on the mass used in the proof of the existence in Theorem 2.4 by the following uniform estimate $\left\|f(t, \cdot) \psi_{0, \beta}\right\|_{M^{1}} \leqslant\left\|f_{\text {in }} \psi_{0, \beta}\right\|_{M^{1}}<\infty$. 
Remark 2.6. The main new results in Theorem 2.4 is the moment estimates (2.15) and (2.16). These ones are based on a trick first introduced in [13]. Estimate (2.15) establishes an instantaneous regularisation effect of the coagulation equation near the origin while (2.16) gives a large time asymptotic behaviour of the generic solutions and shows that these ones have the same behaviour as the self-similar solutions; that is therefore a first validation of (1.13) in the very rough sense of moment estimates.

We extend the previous existence result to the Cauchy problem (1.1), (1.2), with source term $s$ and initial data $f_{\text {in }}$ in a suitable set of measures.

Definition 2.7. We say that a measure $f$ on $[0, \infty) \times[0, \infty)$ is a (global) weak solution to the Cauchy problem (1.1), (1.2) of the coagulation equation with source term $0 \leqslant s \in L^{\infty}\left(0, \infty ; \dot{M}_{1}^{1}\right)$ and initial datum $0 \leqslant f_{\text {in }} \in \dot{M}_{1}^{1}$, if

$$
f \in C\left(\left[0, \infty\left[; \dot{M}_{1}^{1} \text {-weak }\right) \cap L_{\text {loc }}^{1}\left(\left[0, \infty\left[; \mathcal{X}_{a}\right),\right.\right.\right.\right.
$$

and for any $\varphi \in C_{c}^{1}\left(\mathbb{R}_{+}^{2}\right)$

$$
\int_{0}^{\infty} \varphi(0, y) f_{\text {in }}(\mathrm{d} y)-\int_{0}^{\infty} \int_{0}^{\infty} \partial_{t} \varphi f(t, \mathrm{~d} y) \mathrm{d} t=\int_{0}^{\infty}\langle\mathcal{Q}(f(t, \cdot)), \varphi(t, \cdot)\rangle \mathrm{d} t+\int_{0}^{\infty} \int_{0}^{\infty} \varphi s(t, \mathrm{~d} y) \mathrm{d} t .
$$

Theorem 2.8. Assume $\alpha, \beta \in[-1,1], \lambda \in[0,1)$ and $\alpha>\beta-1$. For any $0 \leqslant f_{\text {in }} \in \dot{M}_{1}^{1}$ and $0 \leqslant s \in L^{\infty}\left(0, \infty ; \dot{M}_{1}^{1} \cap\right.$ $\left.\dot{M}_{\xi}^{1}\right)$, with $\xi$ continuous, $\xi(y) \geqslant y$ on $(0, \infty)$ and $\xi(y) / y \rightarrow \infty$ when $y \rightarrow \infty$, there exists at least one weak measure valued solution to the Cauchy problem (1.1), (1.2) such that $f$ conserves the mass

$$
M_{1}(t)=M_{1}(0)+\int_{0}^{t} S(\tau) \mathrm{d} \tau \quad \forall t \geqslant 0, S(t):=\int_{0}^{\infty} y s(t, \mathrm{~d} y) .
$$

Moreover, all the mass of $f(t, \cdot)$ is contained in the particles phase (not in the dust phase), more precisely, for any (small) time $t_{0}>0$

$$
t \mapsto M_{(1+\lambda) / 2}(t) \in L^{\infty}\left(\left[t_{0}, \infty\right)\right) .
$$

Remark 2.9. Since $(1+\lambda) / 2 \in[0,1)$, the estimate (2.20) shows that the density function $y f(t, \mathrm{~d} y)$ does not charge the origin for any $t>0$, which precisely means that no mass is contained in the dust phase. It is again a manifestation of the regularisation property of the coagulation equation as mentioned in Remark 2.5. Estimate (2.20) holds in particular in the case of a pure dust source, that is $s$ such that $y s(t, \mathrm{~d} y)=\sigma(t) \delta_{y=0}$ with $\sigma \in L^{\infty}(0,+\infty)$, and it means that all the dust is instantaneously transformed in particles.

We consider now the self similar solutions.

Definition 2.10. Assume $\lambda<1$ and define $J_{a}=I_{a} \cup[1, \infty)$. We say that a function $G: \mathbb{R}_{+} \rightarrow \mathbb{R}_{+}$is a self-similar profile of mass $\rho$ if

$$
G \in \mathcal{Y}_{a}:=\bigcap_{k \in J_{a}} \dot{L}_{k}^{1}, \quad M_{1}(G)=\rho,
$$

and $G$ is a solution of (1.18) in the following weak sense

$$
\forall \varphi \in C_{c}^{1}\left(\mathbb{R}_{+}\right) \quad \int_{0}^{\infty} G D^{*} \varphi \mathrm{d} y+(1-\lambda)\langle\mathcal{Q}(G), \varphi\rangle=0,
$$

where

$$
D^{*} \varphi(y)=2 \varphi(y)-\partial_{y}(y \varphi)=-y^{2} \partial_{y}\left(\frac{\varphi}{y}\right) .
$$


For $\alpha \geqslant 0$, we will need another representation of the profile equation (1.9) which is given by the following.

Lemma 2.11. Assume $\beta \in[0,1)$ and $\alpha \in[-\beta, \beta] \cap[-\beta, 1-\beta)$. A function $G \in \mathcal{Y}_{a}$ is a self-similar profile in the weak sense of Definition 2.10 if, and only if, $G$ satisfies

$$
z^{2} G(z)=(1-\lambda) \mathcal{C}(G)(z) \quad \text { a.e. on }(0, \infty) .
$$

Proof of Lemma 2.11. On the one hand, for a given $G \in \mathcal{Y}_{a}$ and for any $\varphi \in C_{c}^{1}(0, \infty)$, we define $\varphi(y)=y \psi(y) \in$ $C_{c}^{1}(0, \infty)$ and we compute thanks to Definition 2.10 and Lemma 2.1

$$
\begin{aligned}
\left\langle\partial_{y}\left(G y^{2}\right), \psi\right\rangle & =-\left\langle G, y^{2} \partial_{y} \psi\right\rangle=\left\langle G, D^{*} \varphi\right\rangle=(\lambda-1)\langle\mathcal{Q}(G), \varphi\rangle=(\lambda-1)\langle y \mathcal{Q}(G), \psi\rangle \\
& =(1-\lambda)\left\langle\partial_{y} \mathcal{C}(G), \psi\right\rangle .
\end{aligned}
$$

In other words, that means

$$
\partial_{y}\left(G y^{2}\right)=(1-\lambda) \partial_{y} \mathcal{C}(G) \quad \text { in } \mathcal{D}^{\prime}(0, \infty)
$$

On the other hand, we infer from Lemma 2.1 and the assumption $G \in \mathcal{Y}_{a}$ that $L_{v}(G) y^{k} \in L^{\infty}$ for any $k>\beta$ and $v=\alpha, \beta$, which in turn implies $L_{\alpha}(G), L_{\beta}(G) \in L^{1}$, by choosing for instance $k=2$ and $k=(1+\beta) / 2$. Since we have also $y^{\alpha+1} G, y^{\beta+1} G \in L^{1}$, Lemma 4.4 implies $\mathcal{C}(G) \in L^{1}$. We conclude the proof, integrating Eq. (2.24) and using that $y^{2} G, \mathcal{C}(G) \in L^{1}$.

Our last result is a pointwise estimate from above and below near the origin and at infinity of the self similar profiles which improves the estimates established in $[14,16]$, but are still a weak version of the expected asymptotic behaviour (1.14)-(1.17).

Theorem 2.12. Assume $\beta \in[0,1)$ and $\alpha \in[-\beta, \beta] \cap[-\beta, 1-\beta)$.

(1) Assume $\alpha<0$. For any $\rho>0$ there exists at least one self-similar profile of mass $\rho$ such that $G \in C^{\infty}((0, \infty))$ and

$$
\mathrm{e}^{-a y^{\alpha}} \mathbf{1}_{y \leqslant 1}+\mathrm{e}^{-b y} \mathbf{1}_{y \geqslant 1} \leqslant G(y) \leqslant \mathrm{e}^{-A y^{\alpha}} \mathbf{1}_{y \leqslant 1}+\mathrm{e}^{-B y} \mathbf{1}_{y \geqslant 1} \quad \forall y \in(0, \infty)
$$

for some constants $a, b, A, B>0$.

(2) Assume $\alpha \geqslant 0$. For any $\rho>0$ there exists at least one self-similar profile of mass $\rho$ such that $G \in C((0, \infty))$ and

$$
\begin{aligned}
& \forall \varepsilon>0, \forall y \in(\varepsilon, \infty) \quad \mathrm{e}^{-b_{\varepsilon} y} \leqslant G(y) \leqslant \mathrm{e}^{-B_{\varepsilon} y} \quad \text { for some constants } b_{\varepsilon}, B_{\varepsilon}>0, \\
& G y^{k} \in L^{\infty}(0,1) \quad \forall k>1+\lambda,
\end{aligned}
$$

and moreover if $\alpha>0$ there holds $G y^{k} \notin L^{\infty}(0,1) \forall k<1+\lambda$.

We refer to [16,14] and the surveys [23,27] for a general discussion about self-similar solutions and for references. Coming back to (1.8) and (1.12) the above theorem shows that we are able to build in the case of a pure Dirac mass initial datum $y f_{\text {in }}=\rho \delta_{y=0}$ a (self-similar) solution $F$ to the coagulation equation which has much more regularity and for which we know very much more accurate asymptotic behaviour than for the one built thanks to Theorem 2.8. The proof of Theorem 2.12 is based on the one hand on the power moment estimates (2.15), (2.16) and on exponential moment estimates in the spirit of $[4,5,44]$ and on the other hand on a new bootstrap regularity argument taking advantage of the formulation (2.24).

\section{Moment estimates for the coagulation equation in self-similar variables}

In this section we establish some new a priori moment estimates on the solutions $g$ to the coagulation equation in self-similar variables (1.18). We show the production of moments of order lower than one and the propagation in time of moments larger than one and exponential powers. As a consequence we will deduce a regularising effect of this equation near the origin. 
Such estimates hold for the coagulation profiles $G$ and provide regularity results for the self similar solutions of (1.1). All these estimates will be established formally for a given solution $g$ to the coagulation equation in selfsimilar variables (1.18) associated to an initial datum $g_{\text {in }} \in \dot{L}_{1}^{1}$ with mass $\rho>0$. More precisely, we will use without justification that for any $\phi$

$$
\frac{\mathrm{d}}{\mathrm{d} t} \int_{0}^{\infty} g \phi \mathrm{d} y=\frac{1}{2} \int_{0}^{\infty} \int_{0}^{\infty} a \Delta_{\phi} g g^{\prime} \mathrm{d} y \mathrm{~d} y^{\prime}+\int_{0}^{\infty} g D^{*} \phi \mathrm{d} y
$$

with

$$
\Delta_{\phi}\left(y, y^{\prime}\right)=\phi^{\prime \prime}-\phi-\phi^{\prime} \quad \text { and } \quad D^{*} \phi=-y^{2} \partial_{y}\left(\frac{\phi}{y}\right) .
$$

Notice that for the sake of simplicity we have removed (thanks to a trivial change of unknown) the term $(1-\lambda)$ in front of the first term in the right-hand side term of (3.1). This identity can either be proved rigorously on a (strong) solution associated to the same equation with truncated coagulation rate and passing to the limit, or the associated inequality (with $\leqslant$ sign) can be proved a posteriori on a given weak solution for any subadditive moment functions $\phi$. We postpone this question to Section 6.

Unless it is explicitly specified, we assume in all this section that $0 \leqslant \beta<1$ and $\alpha \in[-\beta, \beta] \cap[-\beta, 1-\beta)$, in such a way that $\lambda \in[0,1)$, and $g_{\text {in }} \in \dot{L}_{1}^{1}$. We then consider a given solution $g$ to (3.1) for which we obtain several estimates, where the constants only depend on $g_{\text {in }} \in \dot{L}_{1}^{1}$.

As a first consequence of (3.1), taking $\phi(y)=y$, we obtain that $g$ conserves the mass

$$
M_{1}(t) \equiv M_{1}(0)=: \rho \quad \forall t \geqslant 0 .
$$

Lemma 3.1. For any $k \in(\lambda, 1)$, there exists $w_{k}=w_{k}(\lambda) \in(0, \infty)$ such that

$$
\forall t \geqslant 0 \quad N_{k}(t) \leqslant \min \left(\frac{w_{k}}{t \wedge 1}, N_{k}(0) \vee w_{k}\right), \quad \text { with } N_{k}(t):=\int_{0}^{\infty} g(t, y)(y \wedge 1)^{k} \mathrm{~d} y .
$$

Moreover, there exists $\eta:(0, \infty) \rightarrow(0, \infty)$ (which only depends on $\left.g_{\text {in }}\right)$ such that $\eta(\varepsilon) \rightarrow 0$ when $\varepsilon \rightarrow 0$ and

$$
\forall t \geqslant 0 \quad \int_{0}^{\varepsilon} y g(t, y) \mathrm{d} y \leqslant \eta(\varepsilon) .
$$

Here and below we define $a \wedge b=\min (a, b), a \vee b=\max (a, b)$, for $a, b \in \mathbb{R}$. The lemma is based on a trick introduced in [13] in order to investigate the gelation phenomenon (when $\lambda>1$ ) which is similar to an idea introduced in [6] in order to deal with elliptic equations with right-hand side Dirac mass. Then, this trick has been used in [24,34] in order to prove similar (but weaker) estimates that those established in Lemma 3.1 on the long time asymptotic of solutions $f$ to the coagulation equation when $\lambda \leqslant 1$ (see [23, Proposition 2] for a precise statement). More recently in [16] , these estimates has been taken up again in order to obtain bounds on self-similar profile to the coagulation equation when $\lambda \leqslant 1$ and $\alpha>0$.

Proof of Lemma 3.1. Step 1. Proof of (3.3). First, we define $\phi_{A}(y)=(y \wedge A)^{m}$ for $m \in(0,1]$ and $A>0$, and we compute

$$
-\Delta_{\phi_{A}}\left(y, y^{\prime}\right)= \begin{cases}y^{m}+y^{\prime m}-\left(y+y^{\prime}\right)^{m} & \text { on }\left\{y, y^{\prime} ; y+y^{\prime} \leqslant A\right\}, \\ y^{m}+y^{\prime m}-A^{m} & \text { on }\left\{y, y^{\prime} ; y \leqslant A, y^{\prime} \leqslant A, y+y^{\prime} \geqslant A\right\}, \\ y^{m} & \text { on }\left\{y, y^{\prime} ; y \leqslant A, y^{\prime} \geqslant A\right\}, \\ y^{\prime m} & \text { on }\left\{y, y^{\prime} ; y \geqslant A, y^{\prime} \leqslant A\right\}, \\ A^{m} & \text { on }\left\{y, y^{\prime} ; y \geqslant A, y^{\prime} \geqslant A\right\},\end{cases}
$$

and

$$
D^{*} \phi_{A}(y)=(1-m) y^{m} \mathbf{1}_{y \leqslant A}+A^{m} \mathbf{1}_{y \geqslant A},
$$


from which we get

$$
-\Delta_{\phi_{A}} \geqslant A^{m} \mathbf{1}_{y, y^{\prime} \geqslant A} \text { and } D^{*} \phi_{A} \leqslant \phi_{A} .
$$

We then deduce from (3.1), (3.5) and the lower estimate $a\left(y, y^{\prime}\right) \geqslant\left(y y^{\prime}\right)^{\lambda / 2}$

$$
\frac{\mathrm{d}}{\mathrm{d} t} \int_{0}^{\infty} g \phi_{A} \mathrm{~d} y+\frac{A^{m}}{2}\left(\int_{A}^{\infty} g y^{\lambda / 2} \mathrm{~d} y\right)^{2} \leqslant \int_{0}^{\infty} g \phi_{A} \mathrm{~d} y \quad \forall A>0 .
$$

Next, for a given function $\Phi:[0, \infty) \rightarrow[0, \infty)$ such that $\Phi(0)=0$ and a given $\ell \in \mathbb{R}$, we have, using Fubini's theorem, Cauchy-Schwarz inequality and (3.6),

$$
\begin{aligned}
\left(\int_{0}^{\infty} g(y) y^{\lambda / 2} \Phi(y) \mathrm{d} y\right)^{2} & =\left(\int_{0}^{\infty} \Phi^{\prime}(A) \int_{A}^{\infty} g(y) y^{\lambda / 2} \mathrm{~d} y \mathrm{~d} A\right)^{2} \leqslant K_{0} \int_{0}^{\infty} \Phi^{\prime}(A) A^{\ell}\left(\int_{A}^{\infty} g(y) y^{\lambda / 2} \mathrm{~d} y\right)^{2} \mathrm{~d} A \\
& \leqslant 2 K_{0} \int_{0}^{\infty} \Phi^{\prime}(A) A^{\ell-m}\left(\int_{0}^{\infty} g \phi_{A} \mathrm{~d} y-\frac{\mathrm{d}}{\mathrm{d} t} \int_{0}^{\infty} g \phi_{A} \mathrm{~d} y\right) \mathrm{d} A \\
& \leqslant 2 K_{0}\left(\int_{0}^{\infty} g \Psi \mathrm{d} y-\frac{\mathrm{d}}{\mathrm{d} t} \int_{0}^{\infty} g \Psi \mathrm{d} y\right),
\end{aligned}
$$

where we have set

$$
K_{0}:=\int_{0}^{\infty} \Phi^{\prime}(A) A^{-\ell} \mathrm{d} A \text { and } \Psi(y):=\int_{0}^{\infty} \Phi^{\prime}(A) A^{\ell-m} \phi_{A}(y) \mathrm{d} A .
$$

In other words, we have obtained the following differential inequality

$$
\frac{\mathrm{d}}{\mathrm{d} t} \int_{0}^{\infty} g \Psi \mathrm{d} y+\frac{1}{2 K_{0}}\left(\int_{0}^{\infty} g(y) y^{\lambda / 2} \Phi(y) \mathrm{d} y\right)^{2} \leqslant \int_{0}^{\infty} g \Psi \mathrm{d} y .
$$

Finally, we make the choices

$$
\Phi(y):=\min \left(y^{\lambda / 2+\delta}, 1\right), \quad \ell:=\lambda / 2, m:=\lambda+2 \delta,
$$

with $\delta \in(0,(1-\lambda) / 2]$ and we easily compute

$$
K_{0}=\left(\frac{\lambda}{2}+\delta\right) \int_{0}^{1} A^{\delta-1} \mathrm{~d} A<\infty, \quad \Psi(y)=\left(\frac{\lambda}{2}+\delta\right)\left(\frac{(y \wedge 1)^{\lambda+\delta}}{\lambda+\delta}+\frac{y^{\lambda+2 \delta}}{\delta}\left(y^{-\delta}-1\right) \mathbf{1}_{y \leqslant 1}\right) .
$$

As a consequence, setting $k=\lambda+\delta \in(\lambda, 1)$, there holds for some constant $C \in(0, \infty)$

$$
\frac{1}{C}(y \wedge 1)^{k} \leqslant \Psi(y) \leqslant C(y \wedge 1)^{k} \text { and } \Phi(y) y^{\lambda / 2} \geqslant(y \wedge 1)^{k} .
$$

Gathering (3.8) and (3.10) we obtain, for some constant $K_{1} \in(0, \infty)$, the differential inequality

$$
\frac{\mathrm{d}}{\mathrm{d} t} \int_{0}^{\infty} g \Psi \mathrm{d} y+K_{1}\left(\int_{0}^{\infty} g \Psi \mathrm{d} y\right)^{2} \leqslant \int_{0}^{\infty} g \Psi \mathrm{d} y .
$$

Performing a time integration of this last one, we deduce that

$$
\int_{0}^{\infty} g \Psi \mathrm{d} y \leqslant\left(\frac{\mathrm{e}^{-t}}{\int_{0}^{\infty} g_{\text {in }} \Psi \mathrm{d} y}+K_{1}\left(1-\mathrm{e}^{-t}\right)\right)^{-1}
$$


and (3.3) follows from (3.10) and (3.11).

Step 2. We prove (3.4). On the one hand, defining the subadditive function $\phi_{\varepsilon}(y)=y \mathbf{1}_{y \leqslant \varepsilon}+\varepsilon \mathbf{1}_{y \geqslant \varepsilon}$, so that $\Delta_{\phi_{\varepsilon}} \leqslant 0$ and $D^{*} \phi_{\varepsilon} \leqslant \phi_{\varepsilon}$, we deduce from (3.1)

$$
\frac{\mathrm{d}}{\mathrm{d} t} \int_{0}^{\infty} g \phi_{\varepsilon} \mathrm{d} y \leqslant \int_{0}^{\infty} g \phi_{\varepsilon} \mathrm{d} y
$$

and then

$$
\sup _{t \in[0,1]} \int_{0}^{\infty} g \phi_{\varepsilon} \mathrm{d} y \leqslant \mathrm{e} \int_{0}^{\infty} g_{\text {in }} \phi_{\varepsilon} \mathrm{d} y=: \eta_{1}(\varepsilon) \rightarrow 0
$$

when $\varepsilon \rightarrow 0$ by Lebesgue dominated convergence theorem. On the other hand, for $t \geqslant 1$ we use (3.3) for a given fixed $k \in(\lambda, 1)$ and we get

$$
\int_{0}^{\varepsilon} g y \mathrm{~d} y \leqslant \varepsilon^{1-k} \int_{0}^{\varepsilon} g y^{k} \mathrm{~d} y \leqslant \varepsilon^{1-k} w_{k}=: \eta_{1}(\varepsilon) \rightarrow 0
$$

when $\varepsilon \rightarrow 0$. We obtain (3.4) gathering (3.12) and (3.13).

Corollary 3.2. For any $k \geqslant 1$ there exists $B_{k}=B(k, \rho, \eta)>0$ such that

$$
\forall t \geqslant 0 \quad M_{k}(t) \geqslant B_{k} .
$$

Proof of Corollary 3.2. We write

$$
M_{k}(t) \geqslant \int_{\varepsilon}^{\infty} g y^{k} \mathrm{~d} y \geqslant \varepsilon^{k-1} \int_{\varepsilon}^{\infty} g y \mathrm{~d} y \geqslant \varepsilon^{k-1}\left(\rho-\int_{0}^{\varepsilon} g y \mathrm{~d} y\right) \geqslant \varepsilon^{k-1}(\rho-\eta(\varepsilon)) \geqslant \varepsilon^{k-1} \frac{\rho}{2}
$$

for $\varepsilon$ small enough, thanks to (3.4).

When $\alpha \leqslant 0$, the moment estimates of Lemma 3.1 may be strengthened in the following way (see also [14,16] for very similar results). When $\alpha<0$, it will be still strengthened as stated in Lemma 3.6 taking advantage of the moment estimates established in Lemma 3.4.

Lemma 3.3. Assume $\alpha \leqslant 0$. There exists $A=A(\rho)>0$ such that (3.3) holds with $k=\lambda$.

Proof of Lemma 3.3. We remark (see the proof of [14, Lemma 4.2]) that

$$
a\left(y, y^{\prime}\right)\left(\left(y+y^{\prime}\right)^{\lambda}-y^{\lambda}-\left(y^{\prime}\right)^{\lambda}\right) \leqslant-C_{\lambda}\left(y y^{\prime}\right)^{\lambda}
$$

from which we deduce

$$
\frac{\mathrm{d}}{\mathrm{d} t} M_{\lambda} \leqslant C_{\lambda, 1} M_{\lambda}-C_{\lambda, 2} M_{\lambda}^{2}
$$

for some constants $C_{\lambda, i}>0$ and then (3.3) with $k=\lambda$ follows by time integration.

Lemma 3.4. For any $k>1$, there exists a constant $A_{k}$ such that

$$
\sup _{[0, \infty)} M_{k}(t) \leqslant \max \left(A_{k}, M_{k}(0)\right) \text {. }
$$

Proof of Lemma 3.4. For a given $k>1$, let us define

$$
\Lambda_{k}\left(y, y^{\prime}\right):=\left(y^{\alpha}\left(y^{\prime}\right)^{\beta}+y^{\beta}\left(y^{\prime}\right)^{\alpha}\right)\left(\left(y+y^{\prime}\right)^{k}-y^{k}-\left(y^{\prime}\right)^{k}\right) \geqslant 0 .
$$


For $y>y^{\prime}$, denoting $z=y^{\prime} / y \in(0,1]$, we have for any $\mu \leqslant 1$

$$
\begin{aligned}
\Lambda_{k}\left(y, y^{\prime}\right) & =y^{\lambda+k}\left(z^{\alpha}+z^{\beta}\right)\left((1+z)^{k}-z^{k}-1\right) \leqslant y^{\lambda+k}\left(2 z^{\alpha}\right)\left(C_{k} z\right) \leqslant 2 C_{k} y^{\lambda+k} z^{\mu+\alpha} \\
& \leqslant 2 C_{k}\left[y^{\beta-\mu+k}\left(y^{\prime}\right)^{\mu+\alpha}+\left(y^{\prime}\right)^{\beta-\mu+k} y^{\mu+\alpha}\right]=: \bar{\Lambda}_{k}\left(y, y^{\prime}\right) .
\end{aligned}
$$

for a constant $C_{k}>0$. Therefore, since $\Lambda_{k}$ and $\bar{\Lambda}_{k}$ are symmetric functions, the inequality $\Lambda_{k}\left(y, y^{\prime}\right) \leqslant \bar{\Lambda}_{k}\left(y, y^{\prime}\right)$ holds for any $y, y^{\prime} \geqslant 0$. We then deduce from (3.1) with $\phi=y^{k}$, the following differential inequality

$$
\frac{\mathrm{d}}{\mathrm{d} t} M_{k} \leqslant C_{k} M_{k+\beta-\mu} M_{\alpha+\mu}-(k-1) M_{k} \text { for any } \mu \leqslant 1 .
$$

Making the choice $\mu:=\beta+\min \left(\frac{k-1}{2}, \frac{1-\lambda}{2}, 1-\beta\right) \in(0,1]$, we obtain

$$
\frac{\mathrm{d}}{\mathrm{d} t} M_{k} \leqslant C_{k} M_{k_{1}} M_{k_{2}}-(k-1) M_{k}
$$

with $k_{1}:=k+\beta-\mu=k-\min \left(\frac{k-1}{2}, \frac{1-\lambda}{2}, 1-\beta\right) \in(1, k), k_{2}:=\alpha+\mu=\lambda+\min \left(\frac{k-1}{2}, \frac{1-\lambda}{2}, 1-\beta\right) \in(\lambda, 1]$. Finally, using the Hölder inequality $M_{k_{1}} \leqslant M_{1}^{1-\theta} M_{k}^{\theta}$, with $\theta \in(0,1)$, we deduce

$$
\frac{\mathrm{d}}{\mathrm{d} t} M_{k} \leqslant C_{1} M_{k}^{\theta} M_{k_{2}}-C_{2} M_{k}
$$

By Lemma 3.1 we have $M_{k_{2}} \in L^{1}+L^{\infty}$. Actually, if $k_{2}=1, M_{k_{2}}=\rho$. If on the other hand, $k_{2} \in(\lambda, 1)$, there is $k_{3}$ such that $\lambda<k_{3}<k_{2}<1$. Then, $M_{k_{2}} \leqslant M_{1}^{\delta} M_{k_{3}}^{1-\delta}$ for some $\delta>0$ and using the estimate (3.3) for $M_{k_{3}}$ we deduce $M_{k_{2}} \in L^{1}+L^{\infty}$. A straightforward integration gives

$$
M_{k}(t)^{1-\theta} \leqslant M_{k}(0)^{1-\theta}+C_{1}(1-\theta)\left\{\frac{\left\|h_{\infty}\right\|_{\infty}}{C_{2}(1-\theta)\left(1-\mathrm{e}^{-C_{2}(1-\theta) t}\right)+\int_{0}^{t} h_{1}(s) \mathrm{d} s}\right\}
$$

where we have introduced the decomposition $M_{k_{2}}=h_{1}+h_{\infty}, h_{1} \in L^{1}, h_{\infty} \in L^{\infty}$.

Corollary 3.5. Assume $g_{\text {in }} \in \dot{L}_{m}^{1}$ for some $m>1$. For any $k \leqslant 1$ there exists $B_{k}=B\left(k, \rho, M_{m}(0)\right)>0$ such that

$$
\forall t \geqslant 0 \quad M_{k}(t) \geqslant B_{k} .
$$

Proof of Corollary 3.5. First, thanks to Lemma 3.4, we have

$$
\sup _{t \geqslant 0} M_{m}(t) \leqslant C
$$

for some constant $C \in(0, \infty)$. Next, using the decomposition

$$
\rho \equiv M_{1}(t) \leqslant R^{1-k} \int_{0}^{R} y^{k} g \mathrm{~d} y+R^{1-m} \int_{R}^{\infty} g y^{m} \mathrm{~d} y \leqslant R^{1-k} M_{k}(t)+R^{1-m} C
$$

with $R$ large enough in such a way that $R^{1-m} C \leqslant \rho / 2$, we get (3.19) with $B_{k}=\rho / 2 R^{k-1}$.

Lemma 3.6. Assume $\alpha<0$ and $g_{\text {in }} \in \dot{L}_{1}^{1} \cap \dot{L}_{m}^{1}$ for some $m>1$.

(1) For any $k \leqslant 0$ there exists $w_{k}=w_{k}\left(\rho, M_{m}(0)\right) \in(0, \infty)$ such that

$$
\forall t \geqslant 0 \quad M_{k}(t) \leqslant \max \left(w_{k}, M_{k}(0)\right) .
$$

(2) More precisely, there exists $A=A\left(\rho, M_{m}(0), a\right) \in(0, \infty)$ and $B_{\text {in }}=B_{\text {in }}\left(g_{\text {in }}\right)$ such that

$$
\forall t \geqslant 0 \quad \int_{0}^{\infty} g(t, y) \mathrm{e}^{(1 \wedge t) y^{\alpha} / A} \mathrm{~d} y \leqslant \min \left(\frac{A}{1 \wedge t}, B_{\text {in }}\right)
$$

with $B_{\text {in }}<\infty$ if (and only if) $g_{\text {in }} \mathrm{e}^{r_{\text {in }} y^{\alpha}} \in L^{1}$ for some $r_{\text {in }}>0$. 
Proof of Lemma 3.6. Step 1. Proof of (3.20). First, for $k \leqslant 0$, we have

$$
a\left(y, y^{\prime}\right)\left(\left(y+y^{\prime}\right)^{k}-y^{k}-\left(y^{\prime}\right)^{k}\right) \leqslant-y^{\alpha+k}\left(y^{\prime}\right)^{\beta}-\left(y^{\prime}\right)^{\alpha+k} y^{\beta}
$$

and we deduce from (3.1) written for $\phi=y^{k}$

$$
\frac{\mathrm{d}}{\mathrm{d} t} M_{k} \leqslant(1-k) M_{k}-M_{\alpha+k} M_{\beta} \leqslant(1-k) M_{k}-c_{*} M_{k}^{1-\frac{\alpha}{1-k}}
$$

with $c_{*}=c_{*}\left(\rho, \beta, \alpha, M_{m}(0)\right):=\inf _{t} M_{\beta}(t) \rho^{\frac{\alpha}{1-k}}>0$, where we have used the Hölder inequality $M_{k} \leqslant M_{1}^{1-\theta} M_{\alpha+k}^{\theta}$, $\theta=(1-k) /(1-k-\alpha)$ and the fact that $M_{\beta}$ is bounded by below thanks to Corollary 3.5. A straightforward integration gives,

$$
M_{k}(t) \leqslant\left(M_{k}(0)^{\frac{\alpha}{1-k}} \mathrm{e}^{\alpha t}+\left(1-\mathrm{e}^{\alpha t}\right) \frac{c_{*}}{1-k}\right)^{\frac{1-k}{\alpha}}
$$

and (3.20) follows with $w_{k}:=\left(c_{*} /(1-k)\right)^{\frac{1-k}{\alpha}}$.

Step 2. Proof of (3.21). Let now fix $\tau>0$. On the one hand, if for a given $k$, there exists $t \in[0, \tau]$ such that $(1-k) M_{k}(t) \geqslant \frac{c_{*}}{2} M_{k}^{1-\alpha /(1-k)}(t)$, then

$$
M_{k}(t) \leqslant\left(\frac{2(1-k)}{c_{*}}\right)^{\frac{1-k}{-\alpha}}=: C_{k}
$$

with $C_{k}=2^{\frac{1-k}{-\alpha}} w_{k}>w_{k}$, and from Step 1, we deduce that

$$
M_{k}(\tau) \leqslant C_{k} \leqslant\left(\frac{2(1-k)}{c_{*}(-\alpha)}\right)^{\frac{1-k}{-\alpha}} .
$$

On the other hand, if for a given $k$ and for any $t \in[0, \tau]$ we have $(1-k) M_{k}(t) \leqslant \frac{c_{*}}{2} M_{k}^{1-\frac{\alpha}{1-k}}(t)$, then the differential inequality (3.23) reduces to

$$
\frac{\mathrm{d}}{\mathrm{d} t} M_{k} \leqslant-\frac{c_{*}}{2} M_{k}^{1-\frac{\alpha}{1-k}} \text { on }(0, \tau)
$$

which in turns implies (by integration)

$$
\frac{1}{M_{k}^{\frac{-\alpha}{1-k}}(t)} \geqslant \frac{c_{*}}{2} t \frac{-\alpha}{1-k}+\frac{1}{M_{k}^{\frac{-\alpha}{1-k}}(0)} \geqslant \frac{c_{*}}{2} t \frac{-\alpha}{1-k} \quad \text { on }(0, \tau) .
$$

We thus obtain, in particular,

$$
M_{k}(\tau) \leqslant\left(\frac{2(1-k)}{c_{*}(-\alpha) \tau}\right)^{\frac{1-k}{-\alpha}}
$$

Gathering (3.24) and (3.25), we deduce

$$
\forall t \geqslant 0 \quad M_{k}(t) \leqslant\left(\frac{z}{t \wedge 1}(1-k)\right)^{\frac{1-k}{-\alpha}},
$$

with $z:=2 /\left(-c_{*} \alpha\right)$ independent of $k \leqslant 0$. We deduce from Stirling formula that for any $j \in \mathbb{N}$ and any $t \geqslant 0$, there holds

$$
M_{j \alpha}(t) \leqslant\left(\frac{z}{t \wedge 1}(1-j \alpha)\right)^{\frac{1-j \alpha}{-\alpha}} \leqslant\left(\frac{Z}{t \wedge 1}\right)^{j+1} j !
$$

for some constant $Z \in(0, \infty)$. As a consequence, we get for any $r>0$

$$
\int_{0}^{\infty} g \mathrm{e}^{r y^{\alpha}}=\sum_{j=0}^{\infty} \frac{r^{j}}{j !} M_{j \alpha} \leqslant \frac{Z}{t \wedge 1} \sum_{j=0}^{\infty}\left(\frac{r Z}{t \wedge 1}\right)^{j},
$$


and we obtain the first estimate in (3.21) making the choice $A:=2 Z, r:=(t \wedge 1) / A$. For the second estimate in (3.21), we just remark that we may replace in (3.26) the term $Z /(t \wedge 1)$ by $Z_{\text {in }} \in(0, \infty)$ if $g_{\text {in }} \mathrm{e}^{r_{\text {in }} y^{\alpha}} \in L^{1}$.

In the next lemma we obtain an upper exponential bound of the solutions of the coagulation equation in self similar variables (1.18).

Lemma 3.7. For any $\beta \in(-\alpha, 1-\lambda)$ there exists two constants $A=A(\rho) \in(0, \infty), x_{0}=x_{0}(\rho) \geqslant 1$ such that the set

$$
\mathcal{C}_{x}:=\left\{g ; M_{k}(g) \leqslant A \Gamma(k+b) \forall k \in[\min (1,1+\alpha), 2], M_{k}(g) \leqslant A \Gamma(k+b) x^{k-1} \forall k \geqslant 2\right\}
$$

is a invariant domain under the flow of Eq. (1.18) for any $x \geqslant x_{0}$. As a consequence, if $g_{\text {in }} \mathrm{e}^{r_{0} y} \in \dot{L}_{1}^{1}$ for some $r_{0} \in$ $(0, \infty)$ there exists $r_{1} \in(0, \infty)$ such that

$$
\sup _{t \geqslant 0} \int_{0}^{\infty} g(t, y) y \mathrm{e}^{r_{1} y} \mathrm{~d} y<\infty .
$$

Proof of Lemma 3.7. Let us consider

$$
g_{\text {in }} \in \mathcal{Z}_{a}:=\bigcap_{k \geqslant \min (1,1+\alpha)} \dot{L}_{k}^{1} .
$$

By the previous estimates proved above we have $g(t) \in \mathcal{Z}_{a}$ for any $t \geqslant 0$. On the one hand, choosing $\phi(y)=y^{p}$ in (3.1) we get

$$
\frac{\mathrm{d}}{\mathrm{d} t} M_{p}+(p-1) M_{p}=\frac{1}{2} \int_{0}^{\infty} \int_{0}^{\infty} a\left(\left(y^{\prime \prime}\right)^{p}-y^{p}-\left(y^{\prime}\right)^{p}\right) g g^{\prime} \mathrm{d} y \mathrm{~d} y^{\prime} .
$$

In order to estimate the right-hand side of (3.27) we use the following lemma, proved by Bobylev, Gamba and Panferov in [5, Lemma 2].

Lemma 3.8. Assume that $p>1$, and let $k_{p}$ denote the integer part of $\frac{p+1}{2}$. For any $y, y^{\prime}>0$ the following inequalities hold

$$
\sum_{k=1}^{k_{p}-1}\left(\begin{array}{l}
p \\
k
\end{array}\right)\left(y^{k}\left(y^{\prime}\right)^{p-k}+y^{p-k}\left(y^{\prime}\right)^{k}\right) \leqslant\left(\left(y^{\prime \prime}\right)^{p}-y^{p}-\left(y^{\prime}\right)^{p}\right) \leqslant \sum_{k=1}^{k_{p}}\left(\begin{array}{l}
p \\
k
\end{array}\right)\left(y^{k}\left(y^{\prime}\right)^{p-k}+y^{p-k}\left(y^{\prime}\right)^{k}\right),
$$

where $\left(\begin{array}{l}p \\ k\end{array}\right)$ stands for the generalised binomial coefficient.

We deduce from (3.27) and Lemma 3.8

$$
\frac{\mathrm{d}}{\mathrm{d} t} M_{p}+(p-1) M_{p} \leqslant S_{p}
$$

with

$$
S_{p}:=\sum_{k=1}^{k_{p}}\left(\begin{array}{l}
p \\
k
\end{array}\right)\left(M_{k+\alpha} M_{p-k+\beta}+M_{k+\beta} M_{p-k+\alpha}\right) .
$$

Let define $z_{k}$ and $Z_{p}$ by

$$
M_{k}=\Gamma(k+b) z_{k}, \quad Z_{p}:=\max _{k=1, \ldots, k_{p}}\left(z_{k+\alpha} z_{p-k+\beta}, z_{k+\beta} z_{p-k+\alpha}\right) .
$$

In order to estimate $S_{p}$, we use the following lemma proved in [5, Lemma 4].

Lemma 3.9. There exists a constant $C_{0}$ such that

$$
S_{p} \leqslant C_{0} \Gamma(p+2 b+\lambda) Z_{p} \quad \forall p>1 .
$$


Gathering (3.28) and Lemma 3.9 we obtain the following differential inequality on $z_{p}$

$$
\frac{\mathrm{d}}{\mathrm{d} t} z_{p}+(p-1) z_{p} \leqslant C_{0} \frac{\Gamma(p+b+(\lambda+b))}{\Gamma(p+b)} Z_{p},
$$

from which we deduce by classical properties of the Gamma function

$$
\frac{\mathrm{d}}{\mathrm{d} t} z_{p}+(p-1) z_{p} \leqslant C_{1} p^{\lambda+b} Z_{p} \quad \forall p>1
$$

Thanks to Lemmas 3.3 and 3.4 we know that there exists $A \in(0, \infty)$ such that

$$
\sup _{t \geqslant 0} z_{k}(t) \leqslant A \quad \forall k \in[\min (1,1+\alpha), 2] .
$$

Let fix $p_{0} \geqslant 2$ such that $p-1 \geqslant C_{1} A p^{\lambda+b}$ for any $p \geqslant p_{0}$. Thanks to Lemma 3.4 again we may find $x_{0} \geqslant 1$ such

$$
\sup _{t \geqslant 0} z_{k}(t) \leqslant A x_{0}^{k-1} \quad \forall k \in\left[2, p_{0}\right] .
$$

We aim to prove that for any $x \geqslant x_{0}$ and any $k \geqslant p_{0}$

$$
z_{k}(0) \leqslant A x^{k-1} \quad \text { implies } \quad \sup _{t \geqslant 0} z_{k}(t) \leqslant A x^{k-1} .
$$

We argue by induction establishing (3.32) successively on any interval $\left[\min (1,1+\alpha), p_{0}+j(1-\beta)\right]$ when $j \in \mathbb{N}^{*}$. Assume then that (3.32) holds on $\left[\min (1,1+\alpha), p_{0}+j(1-\beta)\right]$ for some $j \in \mathbb{N}$ and let consider $p \in\left(p_{0}+j(1-\beta), p_{0}+(j+1)(1-\beta)\right]$. Remarking that $p-k \geqslant p-k_{p} \geqslant 1$ because $p \geqslant 2$ we easily verify that $k+\alpha, p-k+\beta, k+\beta, p-k+\alpha \in\left[\min (1,1+\alpha), p_{0}+j \beta\right]$ for any $k \in\left\{1, \ldots, k_{p}\right\}$. Therefore we may use (3.31) and the recurrence assumption (3.32) for any term $z_{\ell}$ involved in the expression of $Z_{p}$ and we get $Z_{p} \leqslant A^{2} x^{p+\lambda-2} \leqslant A\left(A x^{p-1}\right)$. Inserting this estimate in (3.29) and using the definition of $p_{0}$ we get

$$
\frac{\mathrm{d}}{\mathrm{d} t} z_{p}+(p-1) z_{p} \leqslant(p-1) A x^{p-1} .
$$

Remarking that $\bar{z}_{p}=A x^{p-1}$ is a supersolution for this last equation we deduce that (3.32) holds for $p \in\left(p_{0}+j(1-\right.$ $\left.\beta), p_{0}+(j+1)(1-\beta)\right]$ and then (3.32) holds for any $p \geqslant p_{0}$ by an induction argument. Coming back to the $M_{p}$ functions, we have precisely proved that $\mathcal{C}_{x}$ is an invariant set.

Assume now $g_{\text {in }} \mathrm{e}^{r_{0} y} \in \dot{L}_{1}^{1}$ and compute

$$
\int_{0}^{\infty} g_{\text {in }} y \mathrm{e}^{r_{0} y} \mathrm{~d} y=\sum_{k=0}^{\infty} \int_{0}^{\infty} g \frac{r_{0}^{k}}{k !} y^{k+1} \mathrm{~d} y \leqslant C_{0},
$$

from where we deduce that for any $k \in \mathbb{N}: M_{k+1}(0) \leqslant\left(C_{0} r_{0}\right)(k+1) ! / r_{0}^{k+1}$. Since we may assume without loss of generality that $r \in(0,1]$, the function $y \mapsto \frac{\Gamma(y+b)}{r^{y}}$ is increasing, and we deduce by the Hölder inequality that for any $p \geqslant 1$

$$
M_{p}(0) \leqslant\left(C_{0} r_{0}\right) \frac{\ell_{p} !}{r^{\ell_{p}}} \leqslant\left(C_{0} r_{0}\right) \frac{\Gamma(p+2)}{r^{p+2}} \text { with } \ell_{p}:=[p]+1 .
$$

From the definition of $z_{p}$ we deduce

$$
z_{p}(0) \leqslant\left(C_{0} r_{0}\right) \frac{p(p+1)}{r^{p+2}} \leqslant A x_{1}^{p-1}
$$

for any $p \geqslant 1$ and for some constant $x_{1} \in(1, \infty)$. Choosing $x:=\max \left(x_{0}, x_{1}\right)$ we get from (3.33) and (3.34) that for any $p \geqslant 1$

$$
z_{p}(t) \leqslant A x^{p} ; \quad \forall t \geqslant 0 .
$$

Therefore,

$$
M_{k}(t) \leqslant \Gamma(k+b) A x^{k-1} \quad \forall k \in \mathbb{N}^{*}, \forall t \geqslant 0 .
$$


For $r_{1}<x^{-1}$, we then have

$$
\forall t \geqslant 0 \quad \int_{0}^{\infty} g(t, y) y \mathrm{e}^{r_{1} y} \mathrm{~d} y=\sum_{k=0}^{\infty} \frac{r_{1}^{k}}{k !} M_{k+1}(t) \leqslant A \sum_{k=0}^{\infty}\left(x r_{1}\right)^{k} \frac{\Gamma(k+1+b)}{k !}<\infty .
$$

Putting together the estimates on the solution $g$ to the coagulation equation in self-similar variables obtained in Lemmas 3.1, 3.3, 3.4, 3.6, Corollaries 3.5, 3.2, and using the change of variables (1.19), or more precisely the moment identity (1.20), we obtain the following a priori estimates on the solution $f$ to the coagulation equation in the original variables.

Corollary 3.10. Under the hypothesis $\lambda, \beta \in[0,1)$, a solution $f$ to Eqs. (1.1), (1.2) satisfies

(1) For any $k \in I_{a} \cap[\lambda, 1]$ :

$$
M_{k}(f(t, \cdot)) \leqslant C_{k} t^{\frac{k-1}{1-\lambda}}, \quad \forall t \geqslant 2,
$$

for some positive constant $C_{k}$ only depending on $f_{\text {in }}$ by the mean of its mass $\rho>0$.

(2) Assume $f_{\text {in }} \in \dot{L}_{1}^{1} \cap \dot{L}_{M}^{1}$ with $M>1$. For any $k \in[1, M]$

$$
M_{k}(f(t, \cdot)) \leqslant C_{k} t^{\frac{k-1}{1-\lambda}}, \quad \forall t \geqslant 2,
$$

for some positive constant $C_{k}$ depending on $M_{k}(0)$.

(3) Assume $\alpha \leqslant 0$ and $f_{\text {in }} \in \dot{L}_{1}^{1} \cap \dot{L}_{M}^{1}$ with $M>1$. For any $k \leqslant 0$

$$
M_{k}(f(t, \cdot)) \leqslant C_{k} t^{\frac{k-1}{1-\lambda}}, \quad \forall t \geqslant 2,
$$

for some positive constant $C_{k}$ only depending on $M_{M}(0)$.

(4) Assume $f_{\text {in }} \in \dot{L}_{1}^{1} \cap \dot{L}_{M}^{1}$ with $M>1$. For any $k \leqslant 1$

$$
M_{k}(f(t, \cdot)) \geqslant B_{k} t^{\frac{k-1}{1-\lambda}}, \quad \forall t \geqslant 2,
$$

for some positive constant $B_{k}$ only depending on $M_{M}(0)$.

(5) For any $k \geqslant 1$

$$
M_{k}(f(t, \cdot)) \geqslant B_{k} t^{\frac{k-1}{1-\lambda}}, \quad \forall t \geqslant 2,
$$

for some positive constant $B_{k}$.

\section{Uniform and regularity estimates for self-similar profiles}

In this section we still assume $\beta \in[0,1)$ and $\alpha \in[-\beta, \beta] \cap[-\beta, 1-\beta)$ and thus $\lambda \in[0,1)$. Gathering the results of the preceding section, in particular Lemmas 3.1, 3.3, 3.6, 3.7 and its proof, we see that the set

$$
\mathcal{L C}_{a}:=\left\{g \in \dot{L}_{1}^{1}, M_{1}(g)=\rho, M_{k}(g) \leqslant w_{k} \forall k \in J_{a}\right\} \subset \mathcal{Y}_{a},
$$

where $\left(w_{k}\right)$ is the family of constants defined in these lemmas, is an invariant set under the flow generated by the coagulation equation in self-similar variables (1.18). Moreover, thanks to the proofs of Lemmas 3.6 and 3.7 there exist some constants $r_{\alpha}, r_{1}, w_{\infty} \in(0, \infty)$ such that for any $g \in \mathcal{L C}_{a}$ we have

$$
\int_{1}^{\infty} g(y) \mathrm{e}^{r_{1} y} \mathrm{~d} y+\int_{0}^{1} g(y) y \mathrm{e}^{r_{\alpha} y^{\alpha}} \mathrm{d} y \leqslant w_{\infty}
$$

We now define

$$
\mathcal{M C}_{a}^{+}:=\left\{g \in \mathcal{D}^{\prime}(0, \infty), g \geqslant 0, M_{1}(g)=\rho, M_{k}(g) \leqslant w_{k} \forall k \in I_{a}\right\} .
$$


This is also an invariant set under the flow generated by Eq. (1.18), which furthermore is compact in the weak sense of Radon measures. Let emphasise that the coagulation operator $\mathcal{Q}$ is well defined on $\mathcal{M C}_{a}^{+}$, thanks to Lemma 2.1, and therefore in the sense of Definition 2.10 (that we may extend straightforwardly to a measure framework).

Thanks to the invariant and compactness properties of $\mathcal{M C}_{a}^{+}$the following existence result of self similar profiles can be proved, see $[14,16]$.

Theorem 4.1. There exists at least one weak self-similar profile $G \in \mathcal{M C}_{a}^{+}$, in the sense of Definition 2.10 (extended to a measure framework), for any given mass $\rho>0$.

The proof of Theorem 4.1 can be performed in two steps. In a first step, for any $\varepsilon>0$, we consider a regularised version $\left(E_{\varepsilon}\right)$ of the coagulation equation in self-similar variables (1.18) for which similar estimates than for (1.18) can be obtained and which furthermore generate a (weakly) continuous semi-group in $L^{1}$. Applying the Brouwer (or Tykonov) fixed point theorem one gets the existence of a stationary solution $0 \leqslant G_{\varepsilon} \in \mathcal{L C}_{a}$. In a second step, we remove the regularisation term in $\left(E_{\varepsilon}\right)$ in the limit $\varepsilon \rightarrow 0$ in such a way to recover Eq. (1.18) keeping the uniform estimate $G_{\varepsilon} \in \mathcal{M C}_{a}^{+}$. We conclude using a stability principle in the spirit of the one used in the proof of Theorem 2.4.

The aim of this section is to prove some further properties of the self-similar profiles $G$ given by Theorem 4.1. In all the remainder of this section, we then fix a self-similar profile $G$ given by Theorem 4.1 (in particular $G$ is just assumed to be a measure), which therefore satisfies the bound conditions (4.1).

Theorem 4.2. Assume $\alpha<0$. Then,

$$
\forall k \in \mathbb{N}, \exists r_{k}>0 \quad \text { such that } \mathrm{e}^{r_{k}\left(y^{\alpha}+y\right)} \partial_{y}^{k} G \in L^{\infty} .
$$

In particular $G \in \mathcal{S}(0, \infty)$ the Schwartz space on $(0, \infty)$.

Proof of Theorem 4.2. Due the bounds on the moments of $G$ and Lemma 2.1 point (iii), we may write the strong formulation (or mild formulation, see [14, Definition 2.4 and Remark 2.5]) of the self-similar equation (2.22)

$$
\partial_{y}\left(y^{2} G\right)=y Q(G) \text { in } \mathcal{D}^{\prime}(0, \infty),
$$

from which we deduce for $\gamma=\alpha, 1$ and $r \geqslant 0$

$$
\partial_{y}\left(y^{2} \mathrm{e}^{r y^{\gamma}} G\right)=\left(\gamma r y^{1+\gamma} G+y Q(G)\right) \mathrm{e}^{r y^{\gamma}},
$$

when this equation makes sense.

We remind that the coagulation operator $Q(G)=Q^{+}(G)-Q^{-}(G)$ is defined thanks to the duality formula (2.12). We remark, using $\left(y^{\prime \prime}\right)^{\gamma} \leqslant y^{\gamma}+\left(y^{\prime}\right)^{\gamma}$ for $\gamma=\alpha$ and $\gamma=1$, that we have for any $\varphi \in C_{b}([0, \infty))$

$$
\left\langle Q^{ \pm}(G), \mathrm{e}^{r y^{\gamma}} \varphi\right\rangle \leqslant\|\varphi\|_{L^{\infty}}\left(\int_{0}^{\infty}\left(y^{\alpha}+y^{\beta}\right) \mathrm{e}^{r y^{\gamma}} \mathrm{d} G(y)\right)^{2},
$$

and $Q(G) \mathrm{e}^{r y^{\gamma}} \in M^{1}$ for $r>0$ small enough thanks to (4.1). Therefore, all the terms involved in (4.4) are bounded measures for $r>0$ small enough, and (4.4) make sense. We deduce that $y^{2} \mathrm{e}^{r y^{\gamma}} G \in B V([0, \infty))$, so that $G$ is in fact a measurable function and satisfies (4.2) for $k=0$.

Next, gathering (4.1) with estimate (4.2) for $k=0$, we easily deduce from the first definition of the coagulation operator (1.3) that $\mathrm{e}^{r y^{\gamma}} Q(G) \in L^{\infty}$, and then from (4.4) that

$$
y^{2} \mathrm{e}^{r y^{\gamma}} \partial_{y} G=\partial_{y}\left(y^{2} \mathrm{e}^{r y^{\gamma}} G\right)-G \partial_{y}\left(y^{2} \mathrm{e}^{r y^{\gamma}}\right) \in L^{\infty}
$$

for $r>0$ small enough. Therefore, $G$ satisfies (4.2) for $k=1$.

Finally, we differentiate the coagulation kernel and, using that $G \mathrm{e}^{r_{0} y^{\alpha}} \in L^{\infty}$, we get

$$
\begin{aligned}
\partial_{y}(Q(G))= & \frac{1}{2} \int_{0}^{y}\left(\partial_{y} a\right)\left(y-y^{\prime}, y^{\prime}\right) G\left(y-y^{\prime}\right) G\left(y^{\prime}\right) \mathrm{d} y^{\prime}-\int_{0}^{\infty}\left(\partial_{y} a\right)\left(y, y^{\prime}\right) G G \mathrm{~d} y \\
& +\frac{1}{2} \int_{0}^{y} a\left(y-y^{\prime}, y^{\prime}\right) \partial_{y} G\left(y-y^{\prime}\right) G\left(y^{\prime}\right) \mathrm{d} y^{\prime}-\int_{0}^{\infty} a\left(y, y^{\prime}\right) \partial_{y} G(y) G\left(y^{\prime}\right) \mathrm{d} y^{\prime} .
\end{aligned}
$$


Then, $\mathrm{e}^{r y^{\gamma}} \partial_{y}(Q(G)) \in L^{\infty}$ for $r>0$ small enough. From elementary differential calculus and Eq. (4.3), we also have

$$
\partial_{y}^{2} G=y^{-2} \partial_{y}^{2}\left(y^{2} G\right)-3 y^{-3} \partial_{y}\left(y^{2} G\right)+6 y^{-2} G=y^{-1} \partial_{y}(Q(G))-3 y^{-2} Q(G)+6 y^{-2} G,
$$

from which we conclude that (4.2) holds for $k=2$. We end the proof using an induction argument.

We give now the estimates that we obtain for the self similar profiles when $\alpha \geqslant 0$. The main difference with the case $\alpha<0$ is that $G$ has no so good moment estimates as in that previous case and thus the self-similar profile equation (1.9) has to be understand in the weak sense.

Theorem 4.3. Assume $\alpha \geqslant 0$. There exists $B>0$ such that

$$
y^{k} G \mathrm{e}^{B y} \in L^{\infty} \text { for any } k>\lambda+1 .
$$

Moreover,

$$
y^{2} G \in C^{0, \theta} \quad \text { for any } \theta \in[0,1-\lambda) .
$$

Proof of Theorem 4.3. The idea is to work with the representations (2.25) and (2.24) thanks to Lemma 2.11 and to use the following result, that we state without proof since it follows from the classical Young inequality.

Lemma 4.4. Let $p \geqslant 1, q \in(1, \infty)$ and define $\theta:=\frac{1}{p}+\frac{1}{q}-1 \in(-1,1)$. Consider $g \in\left(L^{1} \cap L^{q}\right)(0, \infty)$ and $f \in$ $L^{p}(0, \infty)$ if $p>1, f \in M^{1}(0, \infty)$ if $p=1$.

(1) If $\theta>0$, we have $f \star_{z} g \in L^{r}(0, \infty)$ with $r=\theta^{-1} \in[q, \infty)$.

(2) If $\theta=0$, we have $f \star_{z} g \in C_{b}(0, \infty)$.

(3) If $\theta<0$, we have $f \star_{z} g \in C^{0,-\theta}(0, \infty)$.

We assume first that $\alpha=0$ and we split the proof in five steps.

Step 1. We follow the proof of Lemma 2.11. From $y^{k} G \in M^{1}$ for any $k \geqslant \lambda$ and Lemma 2.1 we infer $\left(z^{\beta}+\right.$ $\left.z^{2}\right) L_{\alpha}(G)(z) \in L^{\infty},\left(z^{\alpha}+z^{2}\right) L_{\beta}(G)(z) \in L^{\infty}$, and thus

$$
L_{\alpha}(G) \in L^{q_{1}}, L_{\beta}(G) \in L^{q_{2}} \quad \text { for any } q_{1} \in[1,1 / \beta), q_{2} \in[1,1 / \alpha) .
$$

Thanks to Lemma 4.4, we deduce from $y^{\alpha+1} G, y^{\beta+1} G \in M^{1}$ and (4.7) that $\mathcal{C}(G) \in L^{r}$ for any $r \in[1,1 / \beta)$. Integrating Eq. (2.25) we thus obtain

$$
G y^{2}=(1-\lambda) \mathcal{C}(G) \text { in } \mathcal{D}^{\prime}(0, \infty),
$$

from which we deduce that $G$ is in fact a measurable function and that (2.24) holds.

Step 2. We use now the self-similar profile equation $(2.24),(2.6)$ in order to increase the regularity estimates on the profile $G$ by a bootstrap argument. Let first assume $z^{2} G \in L^{r}$ with $r \geqslant 1$ and recall that $z^{\lambda} G \in L^{1}$. We look for $\theta \in(0,1)$ and $p \in[1, r]$ such that

$$
\left(z^{\alpha+1} G\right)^{p}=\left(z^{2} G\right)^{r \theta}\left(z^{\lambda} G\right)^{1-\theta} \text {. }
$$

Solving this system of equations

$$
(\alpha+1) p=2 r \theta+\lambda(1-\theta) \text { and } p=r \theta+1-\theta,
$$

we see that

$$
p=\left(\frac{1-\alpha}{1-\beta} r+1\right)^{-1}\left(1+\frac{1-\alpha}{1-\beta}\right) r, \quad \theta=\frac{1-\beta}{1-\beta+r(1-\alpha)}
$$

satisfy (4.8). By Hölder inequality in (4.8) we get that $y^{\alpha+1} G \in L^{p}$ for this value of $p \in[1, r]$. Using next Lemma 4.4 as long as

$$
\frac{1}{\phi_{1}(r)}=\left(\frac{1-\alpha}{1-\beta} r+1\right)\left(1+\frac{1-\alpha}{1-\beta}\right)^{-1} r^{-1}+\alpha-1=\frac{1-\alpha}{2-\lambda}+\alpha-1+\frac{1-\beta}{2-\lambda} \frac{1}{r} \geqslant 0,
$$


we deduce that $\left(y^{\alpha+1} G\right) \star_{z} L_{\beta}(G) \in L^{\phi_{1}(r)}$. We note $\phi_{2}$ the function defined by the above formula interchanging the role of $\alpha$ and $\beta$. Using the same argument for the term $\left(y^{\beta+1} G\right) \star_{z} L_{\alpha}(G)$ and using (2.24), (2.6) we thus obtain that

$$
z^{2} G \in L^{r} \quad \text { implies } \quad z^{2} G \in L^{\phi(r)},
$$

with $\phi(r):=\min \left(\phi_{1}(r), \phi_{2}(r)\right)$ as long as $\phi(r)^{-1} \geqslant 0$. We remark that

$$
\frac{1}{\phi(r)} \leqslant-\eta+\frac{\delta}{r} \quad \text { with } \eta:=1-\beta-\frac{1-\beta}{2-\lambda}>0, \delta:=\frac{1-\alpha}{2-\lambda} \in(0,1) .
$$

Therefore, starting from $z^{2} G \in L^{r_{0}}$ with $r_{0}=1$, we deduce iterating (4.9) that $z^{2} G \in L^{r_{n+1}}$ for any $n \in \mathbb{N}$ with $r_{n+1}=\phi\left(r_{n}\right)$ as long as $\phi\left(r_{n}\right)^{-1} \geqslant 0$. Thanks to (4.10), we have

$$
\frac{1}{\phi\left(r_{n}\right)} \leqslant \delta^{n}-\frac{1-\delta^{n}}{1-\delta} \eta
$$

and the right-hand side term becomes negative for any $n$ larger than a given integer $N=N(\delta, \eta)$. We then have $z^{2} G \in L^{\infty}$ in less than $N$ iteration steps.

Step 3. We next assume $z^{k} G \in L^{\infty}$ with $\lambda+1 \leqslant k \leqslant 2$. From $z^{k} G \in L^{\infty}$ and

$$
\int_{0}^{\infty}\left(z^{\theta} G\right)^{p} \mathrm{~d} y \leqslant\left\|z^{(p \theta-\lambda) /(p-1)} G\right\|_{L^{\infty}}^{p-1}\left\|z^{\lambda} G\right\|_{L^{1}},
$$

we deduce that $z^{\theta} G \in L^{p}$ with $p=(k-\lambda) /(k-\theta)$ for any $\theta \in(1+\lambda, 2)$. Therefore $z^{\theta} G \in L^{(1 / \alpha)^{\prime}}$ for $\theta$ such that

$$
\frac{k-\theta}{k-\lambda}=1-\alpha \quad \text { or equivalently } \quad \theta=k \alpha+\lambda(1-\alpha) .
$$

Since $\theta \leqslant 1+\alpha$, we infer from Lemma 4.4 that

$$
z^{-2}\left(y^{\alpha+1} G\right) \star_{z} L_{\beta}(G) \leqslant z^{\alpha-1-\theta}\left(y^{\theta} G\right) \star_{z} L_{\beta}(G) \leqslant z^{-\phi_{1}(k)} C_{k}
$$

with $C_{k}$ a constant and

$$
\phi_{1}(k):=k \alpha+(1+\lambda)(1-\alpha) .
$$

We define $\phi_{2}$ replacing $\alpha$ by $\beta$ in the above expression. Making the same job for the term $\left(y^{\beta+1} G\right) \star_{z} L_{\alpha}(G)$ and using (2.24), (2.6) we thus obtain that

$$
z^{k} G \in L^{\infty} \quad \text { implies } z^{\phi(k)} G \in L^{\infty},
$$

with $\phi(k):=\max \left(\phi_{1}(k), \phi_{2}(k)\right)$. We easily verify that $\phi(k) \in[1+\lambda, 2]$ for any $k \in[1+\lambda, 2]$, that $\phi(1+\lambda)=1+\lambda$,

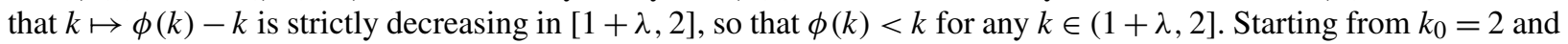
defining the sequence $\left(k_{n}\right)$ by $k_{n+1}=\phi\left(k_{n}\right)$, we deduce from the properties of $\phi$ that $k_{n} \rightarrow 1+\lambda$ when $n \rightarrow \infty$ and therefore (4.5) holds in bounded domains.

Step 4. Interpolating, as in the beginning of step 3, the facts that $z^{k} G \in L^{\infty}$ for any $k>1+\lambda$ and $z^{\lambda} G \in L^{1}$, we find $z^{\alpha+1} G \in L^{p_{2}}$ for any $p_{2} \in[1,1 / \beta)$ and $z^{\beta+1} G \in L^{p_{1}}$ for any $p_{1} \in[1,1 / \alpha)$. Gathering this information with (4.7) we get that $\mathcal{C}(G) \in C^{0, \theta}$ for any $\theta \in(0,1-\lambda)$ thanks to (2.6) and Lemma 4.4, from which (4.6) follows.

Step 5. In order to prove (4.5) with $B>0$, we define $\mathcal{G}(z):=\mathrm{e}^{B y} G(z)$ with $B$ small enough in such a way that $\mathcal{G} \in \mathcal{L C}_{a}$. Eqs. (2.24), (2.6) on $G$ implies that $\mathcal{G}$ satisfies

$$
z^{2} \mathcal{G}(z)=\left(y^{\alpha+1} \mathcal{G}\right) \star_{z} \mathcal{L}_{\beta}(G)+\left(y^{\beta+1} \mathcal{G}\right) \star_{z} \mathcal{L}_{\alpha}(G) \quad \text { a.e. on }(0, \infty),
$$

with

$$
\mathcal{L}_{v}(G)(y):=(1-\lambda) \mathrm{e}^{B y} \int_{y}^{\infty}\left(y^{\prime}\right)^{v} G^{\prime} \mathrm{d} y^{\prime} .
$$

We conclude the proof for the case $\alpha=0$ proceeding along the lines of steps 1 and 2 . When $\alpha>0$ we follow the same steps as for $\alpha=0$. The only difference comes from the fact that, in step 1 , we have $G \in \dot{L}_{k}^{1}$ for any $k \in(\lambda, \infty)$ instead of $G \in \dot{L}_{k}^{1}$ for any $k \in[\lambda, \infty)$. 
Remark 4.5. If we compare (4.6) with Theorem 5.1 we see that (4.6) is near to be the optimal regularity at least for small values of $y$ when $\alpha>0$, because (4.6) implies that $y^{k} G \in L^{\infty}(0,1)$ for any $k>1+\lambda$.

\section{Uniform lower bound for self-similar profiles}

In this section we prove the pointwise lower estimate of the self-similar profiles as stated in Theorem 2.12. It will be a consequence of Theorems 5.1 and 5.4. We start with a profile $G \in \mathcal{Y}_{a}$ whose existence is given by Theorem 4.1 and which is continuous by Theorems 4.2 and 4.3 .

\section{Theorem 5.1.}

(1) For any $\varepsilon>0$, there exists $\delta>0, r<\varepsilon, R>r$, such that $G \geqslant \delta \mathbf{1}_{[r, R]}$.

(2) Assume $\alpha<0$. There exists $a, R>0$ such that $G(y) \geqslant \mathrm{e}^{-a y^{\alpha}}$ on $(0, R)$.

(3) Assume $\alpha>0$. Then $G y^{k} \notin L^{\infty}(0,1)$ for any $k<1+\lambda$.

Proof of Theorem 5.1. Step 1. The general case $\alpha \in \mathbb{R}$. It has already been proved in [16]. We nevertheless present the proof because we will modify it in step 3 below in order to improve the lower estimate when $\alpha>0$. Since $G$ is continuous we just need to prove that $G \not \equiv 0$ on $[0, \varepsilon]$ for arbitrary small $\varepsilon>0$. Assume thus by contradiction that $G \equiv 0$ on $[0, \varepsilon]$ for some $\varepsilon \in(0,1)$. That implies $G \in \dot{L}_{k}^{1}$ for any $k \in \mathbb{R}$ and thus $L_{\alpha}(G), L_{\beta}(G) \in L^{\infty}$. We infer from (2.24)-(2.6) that

$$
z^{2} G(z) \leqslant(1-\lambda)\left(z^{\alpha+1}\left\|L_{\beta}(G)\right\|_{\infty}+z^{\beta+1}\left\|L_{\alpha}(G)\right\|_{\infty}\right) \int_{0}^{z} G(y) \mathrm{d} y \quad \text { on }(0, \infty)
$$

from where we deduce

$$
R^{\prime}(z) \leqslant C R(z) \quad \text { on }(\varepsilon, \infty), \quad R(z):=\int_{0}^{z} G(y) \mathrm{d} y
$$

for some positive constant $C$. Since $R \in C^{1}(0, \infty)$ and $R(\varepsilon)=0$ we obtain $G \equiv 0$ on $(\varepsilon, \infty)$ by the Gronwall lemma. But this is in contradiction with the fact that the mass of $G$ is $\rho>0$.

Step 2. The case $\alpha<0$. On the one hand, $G$ is a strong nonnegative solution of the evolution coagulation equation in self-similar variables (1.18), which means that we can split the $Q^{+}$and the $Q^{-}$terms of the coagulation operator. This equation may then be written as follows:

$$
\partial_{t} G-D G+G \int_{0}^{\infty} a G^{\prime} \mathrm{d} y^{\prime}=\frac{1}{2} \int_{0}^{y} a\left(y-y^{\prime}, y^{\prime}\right) G\left(y-y^{\prime}\right) G\left(y^{\prime}\right) \mathrm{d} y^{\prime} .
$$

We have then

$$
\partial_{t} G-D G+G \ell_{0} \geqslant 0,
$$

where

$$
\ell_{0}(y):=M\left(y^{\alpha}+y^{\beta}\right) \geqslant \int_{0}^{\infty} a G^{\prime} \mathrm{d} y^{\prime}, \quad M:=\max \left(M_{\alpha}(G), M_{\beta}(G)\right) .
$$

On the other hand, any solution $f$ to the equation

$$
\partial_{t} f=D f-\ell_{0} f, \quad f(0)=f_{\text {in }}
$$

is given by the following expression (see [14, Lemma 2.2])

$$
f(t, y)=f_{\text {in }}\left(\mathrm{e}^{t} y\right) \exp \left(2 t-\int_{0}^{t} \ell_{0}\left(y \mathrm{e}^{-(s-t)}\right) \mathrm{d} s\right) \geqslant f_{\text {in }}\left(\mathrm{e}^{t} y\right) \exp \left(-\int_{0}^{t} \ell_{0}\left(y \mathrm{e}^{-s}\right) \mathrm{d} s\right) .
$$


Since $G$ is a time independent function, we then deduce from (5.1) and (5.2) that

$$
\begin{aligned}
G(y) & \geqslant \delta \mathbf{1}_{y \mathrm{e}^{t} \in[r, R]} \exp \left\{-\int_{0}^{t} M\left[\left(y \mathrm{e}^{-s}\right)^{\alpha}+\left(y \mathrm{e}^{-s}\right)^{\beta}\right] \mathrm{d} s\right\} \geqslant \delta \mathbf{1}_{\left\{\mathrm{e}^{-t} r \leqslant y \leqslant \mathrm{e}^{-t} R\right\}} \exp \left\{-\frac{M}{|\alpha|} y^{\alpha}-\frac{M}{\beta}\left(y \mathrm{e}^{-t}\right)^{\beta}\right\} \\
& \geqslant \delta \mathbf{1}_{\left\{\mathrm{e}^{-t} r \leqslant y \leqslant \mathrm{e}^{-t} R\right\}} \exp \left\{-\frac{M}{|\alpha|} y^{\alpha}-\frac{M}{\beta} R^{\beta}\right\}
\end{aligned}
$$

for any $t \geqslant 0$, and we easily conclude.

Step 3. The case $\alpha>0$. Assume, by contradiction, that $G y^{k} \in L^{\infty}$ for some $k \in(1+\beta, 1+\lambda)$. Then, we compute

$$
\begin{aligned}
z^{2} G(z) & \left.=\int_{0}^{z}\left\{\int_{z-y}^{\infty}\left(y^{\prime}\right)^{\alpha-k}\left[G^{\prime}\left(y^{\prime}\right)^{k}\right] \mathrm{d} y^{\prime}\right\} y^{\beta+1-k}\left[G y^{k}\right] \mathrm{d} y+\int_{0}^{z} \int_{z-y}^{\infty} \int_{z}\left(y^{\prime}\right)^{\beta-k}\left[G^{\prime}\left(y^{\prime}\right)^{k}\right] \mathrm{d} y^{\prime}\right\} y^{\alpha+1-k}\left[G y^{k}\right] \mathrm{d} y \\
& \leqslant \frac{\left\|G y^{k}\right\|_{L^{\infty}}^{2}}{k-1-\beta} \int_{0}^{z}\left\{y^{\beta+1-k}(z-y)^{\alpha+1-k}+y^{\alpha+1-k}(z-y)^{\beta+1-k}\right\} \mathrm{d} y \leqslant C_{k} z^{2-(2 k-1-\lambda)},
\end{aligned}
$$

or in other words $G y^{\phi(k)} \in L^{\infty}$ with $\phi(k):=2 k-1-\lambda$. We easily deduce by a finite induction argument that $G y^{k} \in L^{\infty}$ at least for some $k \in(1+\alpha, 1+\beta)$. Remarking that this implies $G y^{\beta} \in L^{1}$, we write now

$$
\begin{aligned}
z^{2} G(z) & =\int_{0}^{z}\left\{\int_{z-y}^{\infty}\left(y^{\prime}\right)^{\alpha-k}\left[G^{\prime}\left(y^{\prime}\right)^{k}\right] \mathrm{d} y^{\prime}\right\} y^{\beta+1-k}\left[G y^{k}\right] \mathrm{d} y+\int_{0}^{z} \int_{z-y}^{\infty} \int_{\left.\left.y^{\prime}\right)^{\beta} G^{\prime} \mathrm{d} y^{\prime}\right\} y^{\alpha+1-k}\left[G y^{k}\right] \mathrm{d} y}^{z} \\
& \leqslant \frac{\left\|G y^{k}\right\|_{L^{\infty}}}{k-1-\alpha}\left(\left\|G y^{k}\right\|_{L^{\infty}}+M_{\beta}(G)\right) \int_{0}^{\infty}\left\{y^{\beta+1-k}(z-y)^{\alpha+1-k}+y^{\alpha+1-k}\right\} \mathrm{d} y \\
& \leqslant C_{k} z^{2-(k-\alpha)} \quad \text { for any } z \in(0,1),
\end{aligned}
$$

or in other words, $G y^{\phi(k)} \in L^{\infty}$ with $\phi(k):=k-\alpha$. We deduce again by an induction argument that $G y^{k} \in L^{\infty}$ for some $k \in(1,1+\alpha)$. That implies $y^{\alpha} G \in L^{1}$ and we have then

$$
z^{1+\alpha} G(z) \leqslant \frac{1}{z^{1-\alpha}} \int_{0}^{z}\left[M_{\beta}(G) y^{\alpha+1}+M_{\alpha}(G) y^{\beta+1}\right] G \mathrm{~d} y \leqslant\left[\frac{M_{\beta}(G)}{z^{1-\alpha}}+\frac{M_{\beta}(G)}{z^{1-\beta}}\right] \int_{0}^{z} z^{1+\alpha} G \mathrm{~d} y .
$$

Defining $R(z):=\int_{0}^{z} y^{1+\alpha} G \mathrm{~d} y$, we have $R \in C^{1}(0, \infty), R(0)=0$ and $R^{\prime}(z) \leqslant C z^{\beta-1} R(z)$ on $(0, \infty)$ with $z^{\beta-1} \in$ $L_{\text {loc }}^{1}([0, \infty))$. By the Gronwall lemma, we get $R \equiv 0$ which is in contradiction with the fact that $G \not \equiv 0$.

We start with two technical lemmas.

Lemma 5.2. For any $R>r>0$, there exists $c_{r, R}>0$ such that

$$
\mathcal{C}\left(\mathbf{1}_{[r, R]}\right) \geqslant c_{r, R} \mathbf{1}_{[r, R+r]} \text {. }
$$

For any $R>4$ and $\gamma \in(0,1 / 2)$, there holds

$$
\mathcal{C}\left(\mathbf{1}_{[1, R]}\right) \geqslant \gamma^{2} \min \left(R^{\alpha}, 1\right) R^{2} \mathbf{1}_{[1,2 R(1-\gamma)]} .
$$

Proof of Lemma 5.2. Distinguishing according to the cases $\alpha \geqslant 0, \alpha<0, r \leqslant 1, r>1, R \leqslant 1$ and $R>1$, there holds

$$
\mathcal{C}\left(\delta \mathbf{1}_{[r, R]}\right)(z) \geqslant \delta^{2} v_{r, R} \int_{0}^{z} \mathbf{1}_{r \leqslant y \leqslant R}\left\{\int_{z-y}^{\infty} \mathbf{1}_{r \leqslant y^{\prime} \leqslant R} \mathrm{~d} y^{\prime}\right\} \mathrm{d} y
$$


with

$$
v_{r, R}:=\min \left(r^{\alpha+1}, r^{\beta+1}\right) \min \left(R^{\alpha}, R^{\beta}, r^{\alpha}, r^{\beta}\right) .
$$

A careful but straightforward computation of the integral term gives

$$
\mathcal{C}\left(\delta \mathbf{1}_{[r, R]}\right)(z) \geqslant \delta^{2} v_{r, R}\left\{\left[(R-r)+\left(z(r+R)-\frac{z^{2}}{2}\right)\right] \mathbf{1}_{[r, R+r]}+\frac{1}{2}(2 R-z)^{2} \mathbf{1}_{[R+r, 2 R]}\right\},
$$

from which we deduce (5.3) and (5.4).

\section{Lemma 5.3.}

(1) Assume that $G \geqslant \delta \mathbf{1}_{[r, R]}$ for some $\delta, r, R>0, R>r$. Then, there exists $C_{r, R}$ such that

$$
G \geqslant \delta^{2} C_{r, R} \mathbf{1}_{[r, R+r]} .
$$

(2) Assume that $G \geqslant \delta \mathbf{1}_{[1, R]}$ for some $\delta>0$ and $R>4$. Then, for any $\gamma \in(0,1 / 2)$ there holds

$$
G \geqslant C \delta^{2} \gamma^{2} R^{-|\alpha|} \mathbf{1}_{[1,2 R(1-\gamma)]}
$$

for some numerical constant $C>0$.

Proof of Lemma 5.3. We prove (5.6). Using Lemma 5.2 we have on $[1,2 R]$

$$
(2 R)^{2} G(z) \geqslant z^{2} G(z)=\mathcal{C}(G)(z) \geqslant \delta^{2} \gamma^{2} \min \left(R^{\alpha}, 1\right) R^{2} \mathbf{1}_{[1,2 R(1-\gamma)]} .
$$

The proof of (5.5) is similar.

Theorem 5.4. For any $r>0$ there exists $b_{r}>0$ such that

$$
G(y) \geqslant \mathrm{e}^{-b_{r} y} \quad \text { on }(r, \infty) \text {. }
$$

Proof of Theorem 5.4. By Theorem 5.1, for $r \in(0,1)$ arbitrary small there exists $\delta^{\prime}>0, R^{\prime}>r$ such that $G \geqslant$ $\delta^{\prime} \mathbf{1}_{\left[r, R^{\prime}\right]}$. Iterating the lower estimate (5.5) we obtain in a finite number of iterative steps that

$$
G \geqslant \delta \mathbf{1}_{[r, R]} \quad \text { with } \delta>0, R>1, r<1 .
$$

We next iterate the lower estimate (5.6) making the choice $\gamma_{i}:=\gamma^{i}$ with $\gamma \in(0,1 / 2)$ to be specified. A straightforward induction argument gives

$$
G(y) \geqslant \prod_{i=0}^{n}\left(C \delta^{2} \gamma_{i}^{2} R_{i}^{-|\alpha|}\right)^{2^{n-i}} \mathbf{1}_{\left[1, R_{n}\right]}(y),
$$

with

$$
R_{n}:=\left[\prod_{i=0}^{n} 2\left(1-\gamma_{i}\right)\right] R .
$$

We then estimate separately each term involved in inequality (5.8) in the three following steps.

Step 1. Using the elementary inequalities $\ln (1-x) \geqslant-2 x$ and $\ln (1+x) \leqslant x$ for $x \in(0,1 / 2)$, we get

$$
2^{n} R \geqslant R_{n}=2^{n}\left[\prod_{j=1}^{n}\left(1-\gamma^{j+1}\right)\right] R \geqslant 2^{n} \mathrm{e}^{-2 \gamma \sum_{j=0}^{\infty} \gamma^{j}} R \geqslant 2^{n} \mathrm{e}^{-4 \gamma} R \geqslant 2^{n-1} R,
$$

for $\gamma$ small enough.

Step 2. We compute the elementary following equivalences

$$
\sum_{i=0}^{n} 2^{n-i} \underset{n \rightarrow \infty}{\sim} 2^{n+1}, \quad \sum_{i=0}^{n} i 2^{n-i} \underset{n \rightarrow \infty}{\sim} 2^{n+1},
$$


from which we deduce

$$
\prod_{i=0}^{n}\left(C \delta^{2} \gamma_{i}^{2} R_{i}^{-|\alpha|}\right)^{2^{n-i}} \geqslant\left(C \delta^{2} R^{-|\alpha|}\right)^{\sum_{i=0}^{n} 2^{n-i}} \prod_{i=0}^{n}\left(\gamma^{2} 2^{-|\alpha|}\right)^{i 2^{n-i}} \geqslant \kappa_{0}^{2^{n}},
$$

with $\kappa_{0}:=\left(C \delta^{2} \gamma^{2} R^{-|\alpha|}\right)^{4}$ (which can be chosen smaller than 1, taking $\gamma$ small enough).

Step 3. Gathering the estimates established in step 1 and step 2, we get

$$
G(y) \geqslant \kappa_{0}^{2^{n}} \mathbf{1}_{\left[1,2^{n-1} R\right]}(y) \geqslant \kappa_{0}^{4 y / R} \mathbf{1}_{\left[2^{n-2} R, 2^{n-1} R\right]}(y) \quad \forall n \geqslant 2,
$$

which in turn implies $G(y) \geqslant \mathrm{e}^{-b_{1} y}$ with $b_{1}=4\left|\ln \kappa_{0}\right| / R$. We conclude gathering this estimate with (5.7).

Proof of Theorem 2.12. It is just enough to gather Theorems 4.1-4.3, 5.1 and 5.4.

\section{Estimates for the coagulation equation in the original variables: proofs of Theorems 2.4 and 2.8}

The main purpose of this section is to prove Theorem 2.4. Therefore we assume in all the following that the hypothesis of Theorem 2.4 are satisfied, namely $\beta \in[0,1], \alpha \in[-1, \beta]$ and $\lambda \in[0,2]$. We start considering a nonnegative initial datum $f_{\text {in }} \in \dot{L}_{1}^{1}$ with $M_{1}\left(f_{\text {in }}\right)=\rho>0$ and we gather some a priori bounds for solutions of the Cauchy problem (1.1), (1.2). Use will be made, without justification, of the following identity for any solution $f$ to the coagulation equation (1.1) and any test function $\phi$ :

$$
\frac{\mathrm{d}}{\mathrm{d} t} \int_{0}^{\infty} f \phi \mathrm{d} y=\frac{1}{2} \int_{0}^{\infty} \int_{0}^{\infty} a \Delta_{\phi} f f^{\prime} \mathrm{d} y \mathrm{~d} y^{\prime}+\int_{0}^{\infty} s \phi \mathrm{d} y,
$$

where $\Delta_{\phi}$ is defined just after (3.1).

We first present an estimate implying the equi-integrability of solutions using a trick introduced in [21] and developed in $[33,24]$.

Lemma 6.1. There exists a real positive function $\Lambda$ such that $\Lambda(0)=0, \Lambda(t) / t \rightarrow \infty$ when $t \rightarrow \infty$, and a constant $C_{0}$ such that

$$
\int_{0}^{\infty} \Lambda(f(t, y)) y \mathrm{~d} y \leqslant C_{0} \quad \forall t \geqslant 0
$$

Proof of Lemma 6.1. On the one hand, from the De La Vallée Poussin lemma, see for instance [22,21], there exists smooth, convex and positive real function $\Lambda$ such that $\Lambda(0)=0, \Lambda(t) / t \rightarrow \infty$ when $t \rightarrow \infty$, and a constant $C_{0}$ such that

$$
\int_{0}^{\infty} \Lambda\left(f_{\text {in }}(y)\right) y \mathrm{~d} y \leqslant C_{0}
$$

On the other hand, we just compute as in the beginning of the proof of (2.4)

$$
\frac{\mathrm{d}}{\mathrm{d} t} \int_{0}^{\infty} \Lambda(f) y \mathrm{~d} y=\int_{0}^{\infty} Q(f) \Lambda^{\prime}(f) y \mathrm{~d} y=\int_{0}^{\infty} \int_{0}^{\infty} a y f f^{\prime}\left(\Lambda^{\prime}\left(f^{\prime \prime}\right)-\Lambda^{\prime}(f)\right) \mathrm{d} y \mathrm{~d} y^{\prime} .
$$

Using the Young inequality $a b \leqslant \Lambda(a)+\Lambda^{*}(b)$ (where $\Lambda^{*}$ stands for the convex conjugate of $\Lambda$ ) and the identity $c \Lambda^{\prime}(c)=\Lambda(c)+\Lambda^{*}\left(\Lambda^{\prime}(c)\right)$ for any $a, b, c \geqslant 0$, we have

$$
f \Lambda^{\prime}\left(f^{\prime \prime}\right)-f \Lambda^{\prime}(f) \leqslant \Lambda(f)+\Lambda^{*}\left(\Lambda^{\prime}\left(f^{\prime \prime}\right)\right)-f \Lambda^{\prime}(f)=\Sigma\left(f^{\prime \prime}\right)-\Sigma(f)
$$

with $\Sigma(a):=a \Lambda^{\prime}(a)-\Lambda(a) \geqslant 0$. We conclude 


$$
\begin{aligned}
\frac{\mathrm{d}}{\mathrm{d} t} \int_{0}^{\infty} \Lambda(f) y \mathrm{~d} y & \leqslant \int_{0}^{\infty} \int_{0}^{\infty} a y f^{\prime}\left(\Sigma\left(f^{\prime \prime}\right)-\Sigma(f)\right) \mathrm{d} y \mathrm{~d} y^{\prime} \\
& \leqslant \int_{0}^{\infty} \int_{0}^{\infty}\left[a\left(y-y^{\prime}, y^{\prime}\right)\left(y-y^{\prime}\right) \mathbf{1}_{y>y^{\prime}}-a\left(y, y^{\prime}\right) y\right] f^{\prime} \Sigma(f) \mathrm{d} y \mathrm{~d} y^{\prime} \leqslant 0,
\end{aligned}
$$

first making the change of variables $\left(y, y^{\prime}\right) \rightarrow\left(y^{\prime \prime}, y\right)$ in the first integral and next noticing that for any $y^{\prime}>0$ the map $z \mapsto z a\left(z, y^{\prime}\right)$ is increasing.

We now give some estimates on the moments of the solution to the coagulation equation (1.1). Most of them are straightforward consequences of the corresponding estimates (3.3), (3.4) and (3.16) for the solution to the coagulation equation in self-similar variables (1.18) using the change of variables (1.19).

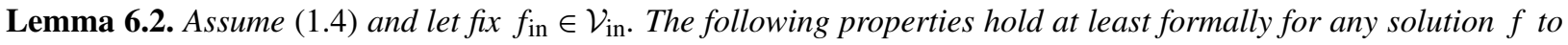
the coagulation equation (1.1), (1.2).

(1) The mass is decreasing in time:

$$
M_{1}(t) \leqslant \rho \quad \forall t \geqslant 0 .
$$

(2) There exists a positive real function $\eta$ such that $\eta(\varepsilon) \rightarrow 0$ when $\varepsilon \rightarrow 0$ and

$$
\int_{0}^{\varepsilon} f(t, y) y \mathrm{~d} y \leqslant \eta(\varepsilon) \quad \forall t \geqslant 0 .
$$

(3) Assume $\lambda \in[0,1)$. For any $k \in I_{a} \cap[\lambda, 1]$ there exists a constant $C_{k}>0$ such that

$$
M_{k}(t) \leqslant \min \left\{\frac{C_{k}}{(t \wedge 1)}, M_{k}(0)\right\} \quad \forall t \geqslant 0 .
$$

(4) Assume $\beta=1$ and $\alpha<0$. There exists $C_{\alpha}>0$ such that

$$
M_{\alpha}(t) \leqslant\left(M_{\alpha}^{\frac{\alpha}{1-\alpha}}(0)+\frac{|\alpha| t}{1+|\alpha|}\right)^{\frac{1-\alpha}{\alpha}} \quad \forall t \geqslant 0 .
$$

(5) Assume $\alpha<0$. There exist $\omega \in(0,1)$ and $C \in(0, \infty)$ such that

$$
M_{1+\alpha}(t) \leqslant C(1+t)^{-\omega} \quad \forall t \geqslant 0 .
$$

Proof of Lemma 6.2. Points (1) and (2) are straightforward consequences of (6.1) using the subadditive test function $\phi(y)=y$ and $\phi(y)=y \mathbf{1}_{0 \leqslant y \leqslant \varepsilon}$. In order to prove point (3), we gather the estimate on $N_{k}$ obtained following the proof of Lemma 3.1 or Lemma 3.3 with the straightforward estimate $M_{k}(t) \leqslant M_{k}(0)$ obtained from (6.1) using the subadditive test function $\phi(y)=y^{k}, k \in I_{a}$.

Proof of (4). Consider $k \leqslant 0$. Using the elementary inequality

$$
a\left(y, y^{\prime}\right)\left(\left(y+y^{\prime}\right)^{k}-y^{k}-\left(y^{\prime}\right)^{k}\right) \leqslant-\left(y^{\alpha+k}\left(y^{\prime}\right)^{\beta}+\left(y^{\prime}\right)^{\alpha+k} y^{\beta}\right)
$$

we deduce (thanks to the Hölder inequality $M_{k} \leqslant M_{k+\alpha}^{\theta} M_{1}^{1-\theta}$ with $\theta:=(k-1) /(k-1+\alpha)$ )

$$
\frac{\mathrm{d}}{\mathrm{d} t} M_{k} \leqslant-M_{\alpha+k} M_{\beta} \leqslant-c M_{k}^{1-\frac{\alpha}{1-k}} M_{\beta}
$$

Assuming now $k=\alpha<0$ and $\beta=1$, we obtain

$$
\frac{\mathrm{d}}{\mathrm{d} t} M_{\alpha} \leqslant-c M_{\alpha}^{1-\frac{\alpha}{1-\alpha}}
$$

from where we deduce (6.6). 
Proof of (5). When $\beta \in[0,1)$ we interpolate (using Hölder inequality) the estimates (6.3) and (6.5) for $k=\lambda$, and we obtain (6.7) with $\omega=-\alpha /(1-\lambda) \in(0,1)$. When $\beta=1$ and therefore by hypothesis $f_{\text {in }} \in \dot{L}_{m}^{1}$ for some $m<1$, we interpolate (6.6) and (6.5) written for $M_{k^{\prime}}$ with $k^{\prime} \in(\max (m, \lambda), 1)$ and we obtain (6.7) with $\omega=\frac{\alpha-1}{\alpha} \frac{1+\alpha-k^{\prime}}{\alpha-k^{\prime}} \in$ $(0,1)$.

We establish now an estimate for superlinear generalised moment.

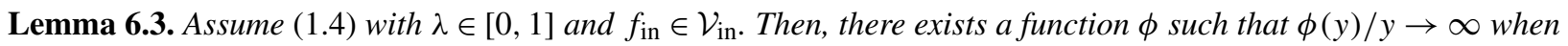
$y \rightarrow \infty$ and for all $T>0$ there exists a positive constant $C \equiv C\left(T, M_{1}(0), M_{m}(0)\right)$ satisfying

$$
\sup _{0 \leqslant t \leqslant T} \mathcal{M}_{\phi}(t) \leqslant C(T) \text {. }
$$

In the proof of Lemma 6.3 we will need the following technical result.

Lemma 6.4. Assume $\lambda \leqslant 1$. For any $\phi$ such that $\phi(y)=y \psi(y), \psi \in C^{1}([0, \infty))$ positive, increasing, $\psi(0)=1$, concave and such that $y \psi^{\prime}(y) \leqslant A(y \wedge 1), \phi(2 y) \leqslant A \phi(y)$ for some constant $A \in(0, \infty)$, there exists a constant $B \in(0, \infty)$ such that

$$
a \Delta_{\phi} \leqslant B\left(K(y) \phi\left(y^{\prime}\right)+\phi(y) K\left(y^{\prime}\right)\right),
$$

with $K(y):=y^{\alpha+1} \mathbf{1}_{y \leqslant 1}+y$.

Proof of Lemma 6.4. Using the decomposition

$$
\Delta_{\phi}=y\left(\psi^{\prime \prime}-\psi\right)+y^{\prime}\left(\psi^{\prime \prime}-\psi^{\prime}\right)
$$

we see that (6.9) reduces to prove

$$
T\left(y, y^{\prime}\right):=y^{\mu+1}\left(y^{\prime}\right)^{\nu}\left[\psi\left(y+y^{\prime}\right)-\psi(y)\right] \leqslant \frac{B}{2}\left(K(y) \phi\left(y^{\prime}\right)+\phi(y) K\left(y^{\prime}\right)\right),
$$

for the two couples $(\mu, v)=(\alpha, \beta)$ and $(\mu, \nu)=(\beta, \alpha)$, or in other words for any couple $(\mu, \nu)$ such that $\mu, \nu \in$ $(-1,1]$ and $\mu+\nu=\lambda$. We estimate $T$ according to different cases.

- When $y^{\prime} \leqslant 1$ or when $v \leqslant 0$, there holds

$$
T \leqslant \psi^{\prime}(y) y^{\mu+1}\left(y^{\prime}\right)^{\nu+1} \leqslant A y^{\mu}(y \wedge 1)\left(y^{\prime}\right)^{\nu+1} \leqslant A y\left[\left(y^{\prime}\right)^{\nu+1} \mathbf{1}_{y^{\prime} \leqslant 1}+y^{\prime}\right]+A\left[y^{\mu+1} \mathbf{1}_{y \leqslant 1}+y\right] y^{\prime},
$$

according to the case $v \leqslant 0$ (and then $\mu \geqslant 0$ ) and to the case $y^{\prime} \leqslant 1$ and $v \geqslant 0$.

- When $1 \leqslant y^{\prime} \leqslant y$ and $v \geqslant 0$, there holds

$$
T \leqslant A y^{\mu}(y \wedge 1)\left(y^{\prime}\right)^{v+1} \leqslant A y^{\lambda}(y \wedge 1) y^{\prime} \leqslant A y y^{\prime} .
$$

- When $1 \leqslant y^{\prime}, y \leqslant y^{\prime}$ and $\nu, \mu \geqslant 0$, there holds

$$
T \leqslant y^{\mu+1}\left(y^{\prime}\right)^{\nu} \psi\left(2 y^{\prime}\right) \leqslant y \mathbf{1}_{y \leqslant 1}\left(y^{\prime}\right)^{\nu} \psi\left(2 y^{\prime}\right)+y^{\lambda} \mathbf{1}_{y \geqslant 1} y^{\prime} \psi\left(2 y^{\prime}\right) \leqslant \frac{A}{2} y \phi\left(y^{\prime}\right) .
$$

- When $1 \leqslant y^{\prime}, v \geqslant 0$ and $\mu \leqslant 0$, there holds

$$
T \leqslant y^{\mu+1}\left(y^{\prime}\right)^{v} \psi\left(2 y^{\prime}\right) \leqslant \frac{1}{2} y^{\mu+1} \phi\left(2 y^{\prime}\right) \leqslant \frac{A}{2}\left[y^{\alpha+1} \mathbf{1}_{y \leqslant 1}+y\right] \phi\left(y^{\prime}\right) .
$$

Proof of Lemma 6.3. From the refined version of De la Vallée Poussin lemma (see for instance [20,35]) there exists $\phi$ satisfying the assumptions of Lemma 6.4 and such that $\mathcal{M}_{\phi}\left(f_{\text {in }}\right)<\infty$. Using Lemma 6.4 and (3.1) there holds

$$
\frac{\mathrm{d}}{\mathrm{d} t} \mathcal{M}_{\phi} \leqslant B \mathcal{M}_{\phi} M_{k} \leqslant B\left(M_{k}+M_{1}\right) \mathcal{M}_{\phi}
$$


with $k=\alpha+1 \in(\lambda, 1)$ if $\alpha<0$ and $k=1$ if $\alpha \geqslant 0$. Since $M_{k} \in L_{\text {loc }}^{1}$ by (6.7) or (6.3) we conclude,

$$
M_{\phi}(t) \leqslant M_{\phi}(0) \exp \left(\int_{0}^{t}\left(M_{k}(s)+M_{1}(s)\right) \mathrm{d} s\right), \quad \forall t>0,
$$

from where (6.8) follows.

Lemma 6.5. Assume $\lambda>1$. There exists a positive constant $C$ such that

$$
\int_{0}^{\infty}\left(\int_{1}^{\infty} y^{\lambda / 2+1 / 2}(\ln y)^{4} f \mathrm{~d} y\right)^{2} \mathrm{~d} t \leqslant C \rho .
$$

Proof of Lemma 6.5. We apply [13, Theorem 2.2] with $\Phi(A):=\left(A^{1 / 2}(\ln A)^{4}\right) \wedge 1$.

Proof of the existence part in Theorem 2.4. It is now classical that in order to prove the existence of solutions to the coagulation equation it is enough to show a "stability result" under "physical estimates" (see for instance [21]). To this end we consider a sequence of functions $\left(f_{n}\right)$ which are weak mass preserving solutions (in the sense of Definition 2.3) to the Cauchy problem (1.1), (1.2) with initial datum $\left(f_{\mathrm{in}, n}\right)$ such that $f_{\mathrm{in}, n} \rightarrow f_{\text {in }}$ weakly in $\dot{L}_{1}^{1}$ and which satisfies, uniformly in $n$, the a priori bounds established in Lemmas 6.1-6.3 and Lemma 6.5, namely:

$$
\begin{aligned}
& M_{1}\left(f_{n}(t)\right) \leqslant \rho \quad \forall t \geqslant 0 ; \\
& \int_{0}^{\varepsilon} f_{n}(t, y) y \mathrm{~d} y \leqslant \eta(\varepsilon) \quad \forall t \geqslant 0 ; \\
& \int_{0}^{\infty} \Lambda\left(f_{n}(t, y)\right) y \mathrm{~d} y \leqslant C \quad \forall t \geqslant 0 ;
\end{aligned}
$$

and for some superlinear function $\phi$ and for some $p \in\left(1, \min \left(\omega^{-1}, 2\right)\right.$ ) (with $\omega \in(0,1)$ defined in Lemma 6.2.5 when $\alpha<0$ and for instance $\omega=1 / 2$ when $\alpha \geqslant 0$ )

$$
\int_{0}^{T}\left(\int_{0}^{\infty} f_{n}(t, y)\left(y^{1+\alpha} \mathbf{1}_{y \leqslant 1}+\phi(y) \mathbf{1}_{y \geqslant 1}\right) \mathrm{d} y\right)^{p} \mathrm{~d} t \leqslant C_{T} \quad \forall T \geqslant 0 .
$$

We aim to prove (and that will establish the announced stability result) that there exist a weak solution $f$ to the Smoluchowski equation (1.1) in the sense of Definition 2.3 and a subsequence $\left(f_{n^{\prime}}\right)$ such that $f_{n^{\prime}} \rightarrow f$ in $C\left([0, \infty) ; L_{\text {loc }}^{1}\right.$-weak). We split now the proof in several steps.

Step 1. Convergence of $\left(f_{n}\right)$. First, from (6.12) and (6.14), there exists $f \in L^{\infty}\left(0, \infty ; \dot{L}_{1}^{1}\right)$ such that (up to the extraction of a sub-sequence)

$$
f_{n} \rightarrow f \quad \text { weakly in } L_{\text {loc }}^{1}\left([0, T] \times \mathbb{R}_{+}\right) \quad \forall T>0 .
$$

From Eq. (6.1) and the estimates (2.9), (6.12) and (6.15) we have that, for any $\phi \in C_{c}^{1}\left(\mathbb{R}_{+}\right)$, the quantity

$$
\frac{\mathrm{d}}{\mathrm{d} t} \int_{0}^{\infty} f_{n}(t, y) \phi \mathrm{d} y=\left\langle\mathcal{Q}\left(f_{n}\right), \phi\right\rangle
$$

is bounded in $L_{\text {loc }}^{p}([0, \infty))$ and thus

$$
f_{n} \rightarrow f \quad \text { in } C\left([0, \infty) ; L_{\text {loc }}^{1}\left(\mathbb{R}_{+}\right) \text {-weak }\right) \text {. }
$$

Step 2. Initial condition. By step 1 we have in particular $f_{n}(0, \cdot) \rightarrow f(0, \cdot)$ in $\mathcal{D}^{\prime}$. Since by hypothesis $f_{\text {in }, n} \rightarrow f_{\text {in }}$, we deduce that $f(0, \cdot)=f_{\text {in }}$. 
Step 3. Passing to the limit in Eq. (2.17). We deduce that for any sequence $\left(\phi_{n}\right)$ such that $\phi_{n}=\phi_{n}\left(t, y, y^{\prime}\right) \rightarrow \phi=$ $\phi\left(t, y, y^{\prime}\right)$ a.e. and $\left\|\phi_{n}\right\|_{L^{\infty}} \leqslant C$, there holds:

$$
\int_{0}^{\infty} f_{n} \phi_{n} \chi^{\prime} \mathrm{d} y \rightarrow \int_{0}^{\infty} f \phi \chi^{\prime} \mathrm{d} y \quad \text { a.e. on } \mathbb{R}_{+}^{2}
$$

with $\chi(y)=y+y^{\min (1,1+\alpha)}$. Writing then

$$
\int_{0}^{T}\left\langle\mathcal{Q}\left(f_{n}\right), \phi\right\rangle \mathrm{d} t=\int_{0}^{T} \int_{0}^{\infty} \int_{0}^{\infty} A_{n} \mathrm{~d} y \mathrm{~d} y^{\prime} \mathrm{d} t
$$

we may pass to the limit $n \rightarrow \infty$ in each subset $\Omega_{i}$ introduced in the proof of Lemma 2.1. We therefore obtain that $f$ is a weak solution in the sense of Definition 2.3.

Proof of the qualitative properties of the solution when $\lambda \in\left[\mathbf{0 , 1}\right.$. From Lemma $6.3, \mathcal{M}_{\phi}(t)$ is bounded on every compact sets of $[0,+\infty)$. We may then pass to the limit in the conservation of mass and then $f$ is also mass conserving. The estimates on the moments of the solution $f$ follow from Corollary 3.10 and Lemma 6.2.

Proof of the gelation property in Theorem 2.4. Assuming $\lambda \in(1,2]$ we show that any weak solution $f \in$ $L^{\infty}\left([0, \infty) ; \dot{L}_{1}^{1}\right)$ does not conserve the mass. Indeed, on the one hand, from [13, Corollary 2.3] the following a posteriori estimate holds

$$
\int_{0}^{\infty}\left(\int_{\varepsilon}^{\infty} f(t, y) y \mathrm{~d} y\right)^{2} \mathrm{~d} t \leqslant C_{\lambda} \varepsilon^{1-\lambda} M_{1}(0)
$$

for some constant $C_{\lambda}$. Let fix $\varepsilon>0$ such that

$$
\int_{0}^{\varepsilon} f(t, y) y \mathrm{~d} y \leqslant \int_{0}^{\varepsilon} f_{\text {in }} y \mathrm{~d} y \leqslant \frac{1}{2} M_{1}(0) .
$$

Assume by contradiction that $M_{1}(t) \equiv M_{1}(0)$. Using the decomposition

$$
\int_{0}^{\infty} f(t, y) y \mathrm{~d} y=\int_{0}^{\varepsilon} f(t, y) y \mathrm{~d} y+\int_{\varepsilon}^{\infty} f(t, y) y \mathrm{~d} y \leqslant \frac{1}{2} M_{1}(0)+\int_{\varepsilon}^{\infty} f(t, y) y \mathrm{~d} y
$$

we get

$$
\frac{1}{2} M_{1}(0) \leqslant \int_{\varepsilon}^{\infty} f(t, y) y \mathrm{~d} y \in L^{2}(0, \infty)
$$

and a contradiction.

Proof of Theorem 2.8. It is very similar to that of Theorem 2.4, where the spaces $\dot{L}_{-}^{1}$ have to be changed to $\dot{M}_{-}^{1}$. Notice nevertheless the following. By the hypothesis on the source term $s$, we are not allowed to use any test function $\phi(y)$ whose behaviour near the origin is $\phi(y) \sim y^{m}$ as $y \rightarrow 0$ with $m<1$. This makes that the regularising effect of the coagulation equation with the source term $s$ is weaker than it was before, without that term, since we only obtain local moments near the origin for $k \in[(1+\lambda) / 2,1)$ instead of $k \in(\lambda, 1)$. The estimate (3.3) of Lemma 3.1 is then modified to estimate (6.17) below. This has only one major consequence in the proof of the theorem. Since we need $M_{1+\alpha} \in L^{1}(0, T)$ in order to define the term $\mathcal{Q}(f)$ in the formulation of weak solution, it is necessary to have $1+\alpha>(1+\lambda) / 2$ or equivalently: $\alpha>\beta-1$. The rest of the arguments being essentially the same we only prove in detail the following lemma from which we easily deduce (2.20). 
Lemma 6.6. Assume $s \in L^{\infty}\left(0, T ; \dot{M}_{1}^{1}\right)$ and $\lambda \in[0,1)$. There exists two positive constants $K_{1}$ and $K_{2}$, independent of $f$ and $f_{\mathrm{in}}$, such that, for all $t \geqslant 0$,

$$
N_{(1+\lambda) / 2}(t) \leqslant\left(N_{(1+\lambda) / 2(0)^{-1}} \mathrm{e}^{-K_{2} t\|S\|_{\infty}}+\frac{K_{1}}{K_{2}\|S\|_{\infty}}\left(\mathrm{e}^{-K_{2} t\|S\|_{\infty}}-1\right)\right)^{-1} .
$$

Proof of Lemma 6.6. We follow the proof of Lemma 3.1. Choosing $\phi=\phi_{A}(y)=(y \wedge A)$ in (6.1) we obtain in the same way that in the proof of (3.6)

$$
\frac{\mathrm{d}}{\mathrm{d} t} \int_{0}^{\infty} f \phi_{A} \mathrm{~d} y+\frac{A}{2}\left(\int_{A}^{\infty} f y^{\lambda / 2} \mathrm{~d} y\right)^{2} \leqslant \int_{0}^{\infty} s \phi_{A} \mathrm{~d} y \leqslant S(t) \quad \forall A>0 .
$$

Next, proceeding along the line of the proof of (3.8) we deduce from (6.18) that for a given function $\Phi:[0, \infty) \rightarrow$ $[0, \infty)$ such that $\Phi(0)=0$ and a given $\ell \in \mathbb{R}$ there holds

$$
\frac{\mathrm{d}}{\mathrm{d} t} \int_{0}^{\infty} f \Psi \mathrm{d} y+\frac{1}{2 K_{0}}\left(\int_{0}^{\infty} f(y) y^{\lambda / 2} \Phi(y) \mathrm{d} y\right)^{2} \leqslant K_{0}^{\prime} S,
$$

where we have defined $K_{0}$ and $\Psi$ thanks to (3.7) with $m=1$ and

$$
K_{0}^{\prime}:=\int_{0}^{\infty} \Phi^{\prime}(A) A^{\ell-1} \mathrm{~d} A .
$$

Finally, we make again the choice (3.9) with now $m=1$ which therefore implies $\delta=(1-\lambda) / 2, \lambda+\delta=(1+\lambda) / 2$ and $K_{0}^{\prime}<\infty$. Thanks to (3.10) we deduce from (6.19) that for some constants $K_{i} \in(0, \infty)$, independent of $f$ and $f_{\text {in }}$, the following differential inequality holds

$$
\frac{\mathrm{d}}{\mathrm{d} t} N_{(1+\lambda) / 2}+K_{1} N_{(1+\lambda) / 2}^{2} \leqslant K_{2} S .
$$

Integrating this differential inequality we obtain (6.17).

\section{Acknowledgements}

The authors gratefully acknowledge the partial support of the European Research Training Network HYKE HPRNCT-2002-00282 during this work. The first author was partially supported by the CICYT under grant BFM200203345. He is grateful to the University of Paris XI Dauphine and CEREMADE for their kind hospitality. We were partially supported by CNRS and UPV through a PIC between the Universidad del Pais Vasco and the Ecole Normale Supérieure. The authors wish to thank the Referee for his very carefully reading of the manuscript. His corrections and remarks have greatly improved the original version of this work.

\section{References}

[1] D.J. Aldous, Deterministic and stochastic models for coalescence (aggregation, coagulation): a review of the mean-field theory for probabilists, Bernoulli 5 (1999) 3-48.

[2] J. Bertoin, The asymptotic behaviour of fragmentation processes, J. Eur. Math. Soc. (JEMS) 5 (4) (2003) $395-416$.

[3] J. Bertoin, Eternal solutions to Smoluchowski's coagulation equation with additive kernel and their probabilistic interpretations, Ann. Appl. Probab. 12 (2) (2002) 547-564.

[4] A.V. Bobylev, Moment inequalities for the Boltzmann equation and applications to the spatially homogeneous problems, J. Statist. Phys. 88 (1997) 1183-1214.

[5] A.V. Bobylev, I. Gamba, V. Panferov, Moment inequalities and high-energy tails for the Boltzmann equations with inelastic interactions, J. Statist. Phys. 116 (5-6) (2004) 1651-1682.

[6] L. Boccardo, T. Gallouët, Nonlinear elliptic equations with right-hand side measures, Comm. Partial Differential Equations 17 (3-4) (1992) 641-655.

[7] S. Cueille, C. Sire, Droplets nucleation and Smoluchovski's equation with growth and injection of particles, Phys. Rev. E 57 (1998) $881-900$.

[8] P.G.J. van Dongen, M.H. Ernst, Cluster size distribution in irreversible aggregation at large times, J. Phys. A 18 (1985) $2779-2793$. 
[9] P.G.J. van Dongen, M.H. Ernst, Solutions of Smoluchowski coagulation equation at large cluster sizes, Physica A 145 (1987) 15.

[10] P.G.J. van Dongen, M.H. Ernst, Scaling solutions of Smoluchowski's coagulation equation, J. Statist. Phys. 50 (1988) $295-329$.

[11] R.L. Drake, A general mathematical survey of the coagulation equation, in: Topics in Current Aerosol Research (Part 2), International Reviews in Aerosol Physics and Chemistry, Pergamon Press, Oxford, 1972, pp. 203-376.

[12] G. Duffa, N.T.-H. Nguyen-Bui, Un modèle de suies, Personal communication, 2002.

[13] M. Escobedo, S. Mischler, B. Perthame, Gelation in coagulation and fragmentation models, Comm. Math. Phys. 231 (2002) 157-188.

[14] M. Escobedo, S. Mischler, M. Rodriguez Ricard, On self-similarity and stationary problem for fragmentation and coagulation models, Ann. Inst. H. Poincaré Anal. Non Linéaire 22 (2005) 99-125.

[15] N. Fournier, J.-S. Giet, On small particles in coagulation-fragmentation equations, J. Statist. Phys. 111 (5-6) (2003) 1299-1329.

[16] N. Fournier, P. Laurençot, Existence of self-similar solutions to Smoluckovski's coagulation equation, Preprint, 2004.

[17] S.K. Friedlander, Smoke, Dust and Haze: Fundamentals of Aerosol Behavior, Wiley, New York, 1979.

[18] I. Jeon, Existence of gelling solutions for coagulation-fragmentation equations, Comm. Math. Phys. 194 (1998) $541-567$.

[19] M. Kreer, O. Penrose, Proof of dynamical scaling in Smoluchowski's coagulation equation with constant kernel, J. Statist. Phys. 75 (1994) 389-407.

[20] Ph. Laurençot, S. Mischler, From the discrete to the continuous coagulation-fragmentation equations, Proc. Roy. Soc. Edinburgh Sect. A 132 (5) (2002) 1219-1248.

[21] Ph. Laurençot, S. Mischler, The continuous coagulation-fragmentation equations with diffusion, Arch. Rational Mech. Anal. 162 (2002) 45-99.

[22] Lê Châu-Hoàn, Etude de la classe des opérateurs $m$-accrétifs de $L^{1}(\Omega)$ et accrétifs dans $L^{\infty}(\Omega)$, Thèse de $3^{\text {ème }}$ cycle, Université de Paris VI, 1977.

[23] P. Laurençot, S. Mischler, On coalescence equations and related models, in: P. Degond, L. Pareschi, G. Russo (Eds.), Modelling and Computational Methods for Kinetic Equations, in: Modeling and Simulation in Science, Engineering and Technology (MSSET), Birkhäuser, 2004, pp. 321-356.

[24] P. Laurençot, S. Mischler, Coagulation and fragmentation equations, in preparation.

[25] M.H. Lee, A survey on numerical solutions to the coagulation equation, J. Phys. A 34 (2001) 10219.

[26] F. Leyvraz, Existence and properties of post-gel solutions for the kinetic equations of coagulation, J. Phys. A 16 (1983) $2861-2873$.

[27] F. Leyvraz, Scaling theory and exactly solved models in the kinetics of irreversible aggregation, Phys. Rep. 383 (2-3) (2003) 95-212.

[28] I.M. Lifshitz, V.V. Slyozov, The kinetics of precipitation from supersaturated solid solutions, J. Phys. Chem. Solids 19 (1961) $35-50$.

[29] A.A. Lushnikov, M. Kulmala, Nucleation burst in coagulating system, Phys. Rev. E 62 (2000) 4932-4939.

[30] E.D. McGrady, R.M. Ziff, "Shattering” Transition in Fragmentation, Phys. Rev. Lett. 58 (1987) 892.

[31] G. Menon, R.L. Pego, Approach to self-similarity in Smoluchowski's coagulation equation, Comm. Pure Appl. Math. 57 (9) (2004) 11971232.

[32] G. Menon, R.L. Pego, Dynamical scaling in Smoluchowski’s coagulation equation: uniform convergence, Preprint, 2003.

[33] S. Mischler, M. Rodriguez Ricard, Existence globale pour l'équation de Smoluchowski continue non homogène et comportement asymptotique des solutions, C. R. Acad. Sci. Paris, Ser. I Math. 336 (2003) 407-412.

[34] S. Mischler, Une introduction aux modèles de coagulation et fragmentation, Notes de cours de DEA, http://www.ceremade.dauphine. $\mathrm{fr} / \sim$ mischler/.

[35] S. Mischler, B. Wennberg, On the spatially homogeneous Boltzmann equation, Ann. Inst. H. Poincaré Anal. Non Linéaire 16 (4) (1999) 467-501.

[36] N. Morgan, C. Wells, M. Kraft, W. Wagner, Modelling nanoparticle dynamics: coagulation, sintering, particle inception and surface growth, Preprint No. 19, Cambridge Center for Computational Chemical Engineering, 2003.

[37] J.R. Norris, Cluster coagulation, Comm. Math. Phys. 209 (2000) 407-435.

[38] J.R. Norris, Smoluchowski's coagulation equation: uniqueness, non-uniqueness and a hydrodynamic limit for the stochastic coalescent, Ann. Appl. Probab. 9 (1999) 78-109.

[39] J.H. Seinfeld, Atmospheric Chemistry and Physics of Air Pollution, John Wiley \& Sons, New York, 1986.

[40] M. Smoluchowski, Drei Vorträge über Diffusion, Brownsche Molekularbewegung und Koagulation von Kolloidteilchen, Physik Z. 17 (1916) 557-599.

[41] M. Smoluchowski, Versuch einer mathematischen Theorie der Koagulationskinetik kolloider Lösungen, Z. Physik. Chemie 92 (1917) 129_ 168.

[42] I.W. Stewart, A global existence theorem for the general coagulation-fragmentation equation with unbounded kernels, Math. Methods Appl. Sci. 11 (1989) 627-648.

[43] I.W. Stewart, A uniqueness theorem for the coagulation-fragmentation equation, Math. Proc. Cambridge Philos. Soc. 107 (1990) $573-578$.

[44] A. Pulvirenti, B. Wennberg, A Maxwellian lower bound for solutions to the Boltzmann equation, Comm. Math. Phys. 183 (1997) 145-160. 